\title{
A factorization approach to next-to-leading-power threshold logarithms
}

\author{
D. Bonocore, ${ }^{a}$ E. Laenen, ${ }^{a, b, c}$ L. Magnea, ${ }^{d}$ S. Melville, ${ }^{e}$ L. Vernazza ${ }^{f}$ and C.D. White ${ }^{e}$ \\ ${ }^{a}$ Nikhef, \\ Science Park 105, NL-1098 XG Amsterdam, The Netherlands \\ ${ }^{b}$ ITFA, University of Amsterdam, \\ Science Park 904, Amsterdam, The Netherlands \\ ${ }^{c}$ ITF, Utrecht University, \\ Leuvenlaan 4, Utrecht, The Netherlands \\ ${ }^{d}$ Dipartimento di Fisica, Università di Torino and INFN, Sezione di Torino, \\ Via P. Giuria 1, I-10125, Torino, Italy \\ e School of Physics and Astronomy, University of Glasgow, \\ Glasgow, G12 8QQ, U.K. \\ ${ }^{f}$ Higgs Centre for Theoretical Physics, School of Physics and Astronomy, University of Edinburgh, \\ Edinburgh, EH9 3JZ, Scotland, U.K. \\ E-mail: d.bonocore@nikhef.nl, Eric.Laenen@nikhef.nl, \\ lorenzo.magnea@unito.it, s.melville.1@research.gla.ac.uk, \\ lvernazz@staffmail.ed.ac.uk, Christopher.White@glasgow.ac.uk
}

ABSTRACT: Threshold logarithms become dominant in partonic cross sections when the selected final state forces gluon radiation to be soft or collinear. Such radiation factorizes at the level of scattering amplitudes, and this leads to the resummation of threshold logarithms which appear at leading power in the threshold variable. In this paper, we consider the extension of this factorization to include effects suppressed by a single power of the threshold variable. Building upon the Low-Burnett-Kroll-Del Duca (LBKD) theorem, we propose a decomposition of radiative amplitudes into universal building blocks, which contain all effects ultimately responsible for next-to-leading-power (NLP) threshold logarithms in hadronic cross sections for electroweak annihilation processes. In particular, we provide a NLO evaluation of the radiative jet function, responsible for the interference of next-to-soft and collinear effects in these cross sections. As a test, using our expression for the amplitude, we reproduce all abelian-like NLP threshold logarithms in the NNLO Drell-Yan cross section, including the interplay of real and virtual emissions. Our results are a significant step towards developing a generally applicable resummation formalism for NLP threshold effects, and illustrate the breakdown of next-to-soft theorems for gauge theory amplitudes at loop level.

Keywords: QCD Phenomenology

ARXIV EPRINT: 1503.05156 


\section{Contents}

1 Introduction 1

2 Organizing NLP threshold logarithms 4

3 The radiative jet function $\quad 12$

3.1 Jet functions for light-like $n^{\mu}-12$

$\begin{array}{ll}3.2 & \text { The radiative jet function at one loop } \\ \end{array}$

4 Application to Drell-Yan production $\quad 17$

$\begin{array}{ll}\text { 4.1 The real-virtual Drell-Yan K-factor at NNLO } & 18\end{array}$

$\begin{array}{lll}4.2 & \text { Reconstructing the K-factor at NLP } & 19\end{array}$

$\begin{array}{lll}\text { 4.2.1 NLP corrections to the phase-space measure } & 19\end{array}$

4.2.2 From tree level to one loop 20

$\begin{array}{lll}\text { 4.2.3 The dressed non-radiative amplitude } & 21\end{array}$

4.2.4 The derivative of the non-radiative amplitude 21

\begin{tabular}{ll}
4.2 .5 & The radiative jet contribution \\
\hline
\end{tabular}

$\begin{array}{lll}4.3 & \text { Discussion } & 23\end{array}$

5 Conclusion $\quad 24$

A The abelian-like real-virtual NNLO Drell-Yan K-factor 26

\section{Introduction}

It has long been known that the emission of soft and collinear gluons causes large corrections in perturbation theory, limiting its range of applicability. At the level of partonic cross sections, these corrections take the form of threshold logarithms of ratios of physical momentum scales, which diverge as the total energy or transverse momentum of emitted gluons (for soft or collinear emission respectively) becomes vanishingly small. Picking a dimensionless variable $\xi$ to measure the kinematic deviation from the threshold region, a differential cross section in this variable has a perturbative expansion of the form

$$
\frac{d \sigma}{d \xi}=\sum_{n=0}^{\infty}\left(\frac{\alpha_{s}}{\pi}\right)^{n} \sum_{m=0}^{2 n-1}\left[c_{n m}^{(-1)}\left(\frac{\log ^{m} \xi}{\xi}\right)_{+}+c_{n m}^{(0)} \log ^{m} \xi+\ldots\right] .
$$

We refer to the leading contributions in this expansion, characterized by the coefficients $c_{n m}^{(-1)}$, as leading-power (LP) threshold logarithms, while the $c_{n m}^{(0)}$ specify next-to-leadingpower (NLP) threshold logarithms, and the ellipsis denotes terms which are suppressed by additional powers of $\xi$. A variety of approaches for describing LP threshold effects exists 
in the literature, such as diagrammatic techniques based on factorization theorems [1-3], approximations using Wilson lines [4, 5], renormalization group arguments [6], dedicated effective field theories [7-10] and path integral techniques [11]. Crucial to all these approaches is the notion of factorization, corresponding to the intuitive idea that long-distance effects in perturbative cross sections must have a universal nature, tied with the wave functions of the scattering states, and it must be possible to disentangle them from the contributions of short-distance high-energy exchanges.

At the level of scattering amplitudes, long-distance effects at leading power manifest themselves in the form of infrared and collinear divergences, which are known to factorize $[12,13]$. For a generic $n$-point gauge theory scattering amplitude, this factorization takes the schematic form

$$
\mathcal{A}_{(n)}=\prod_{i=1}^{n}\left[\frac{J_{i}}{\mathcal{J}_{i}}\right] \cdot \mathcal{S}_{(n)} \cdot \mathcal{H}_{(n)} \cdot
$$

Here the jet functions $J_{i}$ (one for each external parton) contain universal collinear singularities which depend only on the color and spin quantum numbers of the external states, the soft function $\mathcal{S}_{(n)}$ collects all soft singularities, which correlate all partons but do not depend on their energies and spins, and $\mathcal{H}_{(n)}$ is a hard function, which is process dependent and infrared finite. One must finally divide each jet by its eikonal counterpart $\mathcal{J}_{i}$, to correct for the fact that soft and collinear divergences have been double-counted. The factorization in eq. (1.2) leads to exponentiation of soft and collinear factors in terms of a restricted set of anomalous dimensions: color singlet anomalous dimensions are known to three loops, since they can be extracted from the poles of partonic form factors $[14,15]$; the soft anomalous dimension matrix, on the other hand, is known at two loops for both massless and massive partons [16-25]; the development of the necessary techniques to extend these results to the three-loop order and beyond is under way [26-39].

The factorization of leading-power infrared enhancements at amplitude level, embodied by eq. (1.2), ultimately leads to the resummation of LP threshold logarithms for a large class of infrared-safe observables, under mild assumptions concerning the behavior of the associated real radiation [40]. In most cases, current knowledge of the anomalous dimensions allows one to perform this resummation up to $\mathrm{N}^{2} \mathrm{LL}$ or approximate $\mathrm{N}^{3} \mathrm{LL}$ accuracy. Given this increasing theoretical precision, and given the growing demands of current collider experiments, it is natural to attempt to extend existing results to the next set of contributions ${ }^{1}$ in eq. (1.1): indeed, these effects are known to be potentially significant [43-47], as might be expected from the fact that they are still singular as $\xi \rightarrow 0$, albeit integrably so. Furthermore, there are theoretical reasons to expect that it should be possible to organize NLP threshold effects. Indeed, it has been known for a long time that soft gluon radiation effects at NLP can still be expressed in a universal way in terms of the non-radiative matrix element: this is the content of the Low-Burnett-Kroll theorem $[48,49]$. This theorem was extended to the case of massless theories, where collinear effects become important, by Del Duca [50].

\footnotetext{
${ }^{1}$ Recall that another important set of terms can be controlled to all orders, and can be shown to exponentiate in some cases: these are the contributions that have support only on the threshold, proportional to $\delta(\xi)$ if $\xi$ is the threshold variable [41,42]. These terms will not be discussed here.
} 
With these motivations, in recent years several steps were taken towards a deeper understanding of NLP threshold effects and ultimately towards a resummation of NLP threshold logarithms [11, 51-64], including results for a complete resummation of certain towers of NLP logs in specific processes. A fully general resummation prescription, applicable to all processes for which threshold logarithms can be resummed, has however yet to be developed. In particular, one must carefully disentangle the interplay between the soft expansion, which is well under control, and hard collinear effects, which can also be the source of threshold logarithms.

In this paper, we will build on earlier results of refs. [11, 57, 64]. The first of these papers used path integral methods to perform a systematic expansion in powers of the momentum of emitted gluons, thus deriving a generalization of the soft-collinear factorization formula of eq. (1.2), valid up to next-to-soft order. The second paper rederived these results using a diagrammatic approach, and also performed a first check of the formalism, applying it to the case of Drell-Yan production of a color-singlet vector boson. It was shown that the next-to-soft Feynman rules correctly reproduce the known NLP logarithms in the double-real-emission contribution to the $\mathrm{K}$ factor for this process, up to NNLO [65-69]. Whilst refs. $[11,57]$ addressed the factorization and exponentiation properties of next-tosoft effects, the results were limited, as is the earlier work of refs. [48, 49], by the fact that collinear singularities were not properly accounted for. In the case of Drell-Yan production, final state gluons are forced to be (next-to-) soft, so the interplay of soft and collinear enhancements arises only due to the presence of virtual hard-collinear gluons, which distort the emission spectrum of soft gluons at next-to-leading power in the soft expansion. In essence, in the context of eq. (1.2), one must also include the possibility of soft emissions from the jet functions which contain all collinear radiation.

The tools to tackle this problem, and thus to extend the Low-Burnett-Kroll theorem $[48,49]$ to collinear-singular amplitudes, were developed by Del Duca [50], who proposed a generalization of the standard hard-soft factorization, designed to organize the effects of next-to-soft radiation in the massless limit. A further step forward was recently taken by some of us in [64], where NLP threshold corrections to NNLO Drell-Yan production were precisely classified according to their soft or collinear origin, using the method of regions developed in refs. [70-72]. ${ }^{2}$ The results of ref. [64] imply that in the case of electroweak annihilation cross sections the configurations needed to bridge the gap between the soft expansion and the threshold expansion are precisely the collinear-enhanced ones which are taken into account in ref. [50].

Our aim in the present paper is to build on the results of refs. [11, 50, 57, 64], in the light of eq. (1.2), to construct a precise formulation accounting for all NLP threshold logarithms for electroweak annihilation cross sections. With this in mind, we will improve upon the approach of [50] by taking into account the factorization of soft modes (which were included in the hard interaction in ref. [50]), by explicitly taking care of the double counting of soft-collinear singularities, and by discussing the role played by the factorization vectors

\footnotetext{
${ }^{2}$ The method of regions has also been recently applied, in a slightly different context, to characterise threshold effects in Higgs production via gluon fusion up to $\mathrm{N}^{3} \mathrm{LO}$, in refs. [73, 74].
} 
in the factorization of collinear from soft modes. This will lead us to a general expression for the radiative scattering amplitude, similar to that derived in [50], which contains all universal ingredients needed to organize NLP threshold logarithms in the process at hand. We will then test our formalism, once again in the context of Drell-Yan production, by reproducing the known NLP logarithms in the mixed real-virtual contribution to the K factor for this process at NNLO, focussing for simplicity on the abelian-like term proportional to

$C_{F}^{2}$. We will describe how our formalism can be generalized to include purely non-abelian contributions, but we leave more detailed phenomenological analysis to future work.

In parallel to the above developments, a recent body of work has examined the nextto-soft behaviour of gauge and gravity scattering amplitudes from a more formal point of view [75-95], leading to a general theorem for the structure of next-to-soft corrections at tree-level and beyond. Particularly relevant in this regard is the study of ref. [96], which approaches the classification of next-to-soft emissions from the viewpoint of softcollinear effective theory (SCET), including also a detailed discussion of collinear effects. The relationship between this body of work and the approach of this paper has been recently clarified in ref. [97] (see also [98]). Here we will follow up on this, by pointing out the implications of our results for loop corrections to next-to-soft theorems: our results broadly agree with, and substantiate with a concrete example, the arguments of refs. [89, 96], showing that in the presence of collinear singularities next-to-soft theorems for scattering amplitudes require non-trivial corrections at one loop and beyond.

The structure of our paper is as follows. In section 2 we review in detail the modified factorization formula for next-to-soft emissions in the presence of collinear singularities proposed in ref. [50], which we refine in several respects to emphasize the role of soft factors. As in ref. [50], our final expression for the radiative amplitude at next-to-soft level contains a universal function describing the emission of a soft gluon from inside a jet, which we call the radiative jet function. We calculate this function explicitly at one loop in section 3 . In section 4 , we assemble all necessary ingredients in order to reproduce the NLP threshold contributions to the Drell-Yan $K$-factor, providing a strong validation of our expression for the radiative amplitude. We discuss our results in section 5 before concluding. Technical details are presented in an appendix.

\section{Organizing NLP threshold logarithms}

In this section, we discuss in detail the construction of an expression, at the amplitude level, that directly leads to organizing threshold logarithms up to next-to-leading power. As already mentioned in the Introduction, a class of NLP threshold corrections arising from soft gluons at next-to-leading power in the soft expansion was analyzed in refs. [11, 57]. The resulting factorization can describe those processes in which all threshold contributions arise only from soft emissions, but it will fail when collinear singularities are present in addition. This problem is shared by earlier works on next-to-soft corrections, such as the well-known investigation by Low [48], who considered the emission of soft photons from hard scalar emitting particles, and showed how the resulting matrix element can be 
expressed in terms of (derivatives of) the non-radiative amplitude. ${ }^{3}$ This seminal theorem was generalized to fermionic emitters in ref. [49], and the combined result is known as the Low-Burnett-Kroll theorem. This is still not the full story, however, as the results of refs. [48, 49] apply only to particles with a non-vanishing mass $m$, so that one may take the energy $E$ of soft gluons to zero in the parametrically well-defined limit $E / m \rightarrow 0$. One must clearly amend this argument for massless external particles, which in practice involves carefully disentangling collinear singularities (associated with $m \rightarrow 0$ ) from those associated with the soft expansion. This important generalization was achieved by Del Duca [50], who derived an expression for the radiative amplitude valid in the extended region $m^{2} / Q \leq E<m$, where $Q$ is the energy scale associated with the hard interaction. Taking $m / Q$ parametrically to zero, one obtains a modification of eq. (1.2), which is correct (at least in principle) up to next-to-soft level. For the purposes of studying threshold corrections, all these results are necessary, and in this paper we refer to this body of knowledge as the Low-Burnett-Kroll-Del Duca (LBKD) theorem. ${ }^{4}$

Let us begin by reviewing the results of ref. [50], and placing them in the context of eq. (1.2), which was developed in subsequent years. This will enable us to refine the original treatment, in particular explicitly extracting soft effects, studying the dependence on reference vectors defining the collinear region, and addressing the double counting of contributions which are both soft and collinear. We start our analysis by examining in more detail the soft-collinear factorization formula of eq. (1.2) for the specific case of two hard colored particles, which we may write as

$$
\begin{aligned}
\mathcal{A}\left(\frac{Q^{2}}{\mu^{2}}, \alpha_{s}\left(\mu^{2}\right), \epsilon\right)= & \mathcal{H}\left(\left\{p_{i}\right\},\left\{n_{i}\right\}, \alpha_{s}\left(\mu^{2}\right), \epsilon\right) \times \mathcal{S}\left(\left\{\beta_{i}\right\}, \alpha_{s}\left(\mu^{2}\right), \epsilon\right) \\
& \times \prod_{i=1}^{2}\left[\frac{J_{i}\left(p_{i}, n_{i}, \alpha_{s}\left(\mu^{2}\right), \epsilon\right)}{\mathcal{J}_{i}\left(\beta_{i}, n_{i}, \alpha_{s}\left(\mu^{2}\right), \epsilon\right)}\right],
\end{aligned}
$$

Here we work in $d=4-2 \epsilon$ dimensions, and $p_{i}\left(\beta_{i}\right)$ is the four-momentum (four-velocity) of the $i$-th hard particle. Again, $Q$ is the energy scale associated with the hard interaction: for definiteness, we take here $Q^{2}=\left(p_{1}+p_{2}\right)^{2}>0$.

The partonic jet functions appearing in eq. (2.1) are defined by [13]

$$
J\left(p, n, \alpha_{s}\left(\mu^{2}\right), \epsilon\right) u(p)=\left\langle 0\left|\Phi_{n}(\infty, 0) \psi(0)\right| p\right\rangle,
$$

where $\psi(x)$ is a quantum field inserted to absorb the external incoming parton with momentum $p$. The factor $\Phi_{n}$, on the other hand, represents a Wilson line stretching from the absorption point to infinity, along a direction fixed by an auxiliary vector $n^{\mu}$, according to the definition

$$
\Phi_{n}\left(\lambda_{2}, \lambda_{1}\right)=\mathcal{P} \exp \left[i g_{s} \int_{\lambda_{1}}^{\lambda_{2}} d \lambda n \cdot A(\lambda n)\right] .
$$

The presence of the Wilson line ensures that the definition is gauge-invariant. As was remarked in the Introduction, one must further introduce in eq. (2.1) the eikonal counterpart

\footnotetext{
${ }^{3}$ See, for example, appendix $\mathrm{C}$ of ref. [11] for a modern review of this material.

${ }^{4}$ The classification of NLP threshold effects from collinear regions has also been extensively discussed using effective field theory methods in the recent study [96].
} 
of the jet functions, $\mathcal{J}_{i}$, in order to avoid the double counting of soft-collinear contributions. The eikonal jet is defined as

$$
\mathcal{J}\left(\beta, n, \alpha_{s}\left(\mu^{2}\right), \epsilon\right)=\left\langle 0\left|\Phi_{n}(\infty, 0) \Phi_{\beta}(0,-\infty)\right| 0\right\rangle .
$$

Note that eqs. (2.2) and (2.4) apply to incoming particles: for outgoing particles, one would have to reverse the direction of the Wilson lines.

A key point of this discussion is the fact that one must associate with each leg of momentum $p_{i}$ an auxiliary four-vector $n_{i}$. This ensures gauge invariance, but, more interestingly, it can be physically interpreted as providing a means for measuring collinearity with respect to $p_{i}$. The $n_{i}$ 's are 'factorization vectors', and the full amplitude cannot depend on them, much as it cannot depend on the factorization and renormalization scale $\mu$. In fact, singular dependence on $n_{i}$ cancels between each jet and its eikonal counterpart, while non-singular $n_{i}$-dependent terms cancel between the jets and the hard function.

In order not to introduce in the jet functions $J_{i}$ spurious collinear singularities not associated with emissions from the $i$-th hard parton, it is customary in factorization studies [13] to take $n_{i}^{2} \neq 0$. This has the advantage of allowing a cleaner identification of each subset of singular contributions, but it has the drawback of introducing a more complicated functional dependence on the factorization vectors. In contrast, a more physical viewpoint is to consider the $n_{i}$ vectors as standing in to replace the other hard partons in the process: this is what is typically done in effective field theory calculations [96], and was also the approach followed in ref. [64]. This choice leads to much simpler expressions, at the price of a degree of ambiguity in the classification of singular regions. Here, we will follow ref. [50], and define two dimensionless light-like vectors $\hat{n}_{i}$ in directions opposite to $p_{i}$, and such that

$$
\hat{n}_{i}^{2}=0, \quad \hat{n}_{i} \cdot p_{i}=Q .
$$

In the calculations of sections 3 and 4 , it will in fact be more convenient to work with the dimensionful vectors

$$
n_{i}=\frac{Q}{2} \hat{n}_{i}, \quad n_{i} \cdot p=\frac{Q^{2}}{2}
$$

note that for a two-parton process, such as that of eq. (2.1), the definitions of eq. (2.6) can be implemented simply by choosing

$$
n_{1}=p_{2}, \quad n_{2}=p_{1},
$$

an identification that will be useful in section 4 .

Let us now turn to the soft function, which collects infrared singularities associated with the leading (eikonal) term in the momentum expansion of emitted gluons. For twoparton annihilation, it is defined by

$$
\mathcal{S}\left(\beta_{1} \cdot \beta_{2}, \alpha_{s}\left(\mu^{2}\right), \epsilon\right)=\left\langle 0\left|\Phi_{\beta_{2}}(\infty, 0) \Phi_{\beta_{1}}(0,-\infty)\right| 0\right\rangle .
$$

These expressions for the soft and jet functions implicitly define also the process-dependent hard function: for a given process, one defines $\mathcal{H}$ via eq. (2.1), by taking all perturbative 
contributions to the soft and jet functions over to the left-hand side. The hard function then depends on the auxiliary vectors $n_{i}$ precisely in such a way as to cancel the finite parts of their contribution to the jet functions, so that the physical amplitude is independent of each $n_{i}$.

As assembled in eq. (2.1), the double counting of soft-collinear regions is solved by assigning soft-collinear poles to the soft function, and constructing ratios of jets and eikonal jets which contain only hard collinear singularities. An alternative arrangement of the same expression is to assign the soft-collinear poles to the jets, and construct the ratio of the soft function and the eikonal jets, which contains only soft wide-angle radiation. This ratio defines the reduced soft function,

$$
\overline{\mathcal{S}}\left(\frac{\beta_{1} \cdot \beta_{2}}{\beta_{1} \cdot n_{1} \beta_{2} \cdot n_{2}}, \alpha_{s}\left(\mu^{2}\right), \epsilon\right)=\frac{\mathcal{S}\left(\beta_{1} \cdot \beta_{2}, \alpha_{s}\left(\mu^{2}\right), \epsilon\right)}{\prod_{i} \mathcal{J}_{i}\left(\beta_{i} \cdot n_{i}, \alpha_{s}\left(\mu^{2}\right), \epsilon\right)} .
$$

On the left-hand side, we have indicated that the arguments of the soft and jet functions must combine to form a ratio constructed to be invariant under the rescalings $\beta_{i} \rightarrow \lambda_{i} \beta_{i}$. As discussed in detail in $[13,19,99]$, for light-like $\beta_{i}$ this invariance is broken for the soft function alone, as well as for the eikonal jets, due to the presence of collinear divergences in either factor. When the individual factors are combined into the reduced soft function, as in eq. (2.9), collinear poles cancel and the invariance is restored. If, on the other hand, we work with light-like $n_{i}$, the spurious collinear divergences associated with the Wilson lines in the $n_{i}$ directions do not cancel in $\overline{\mathcal{S}}$, so the expected invariance under the rescalings $n_{i} \rightarrow \kappa_{i} n_{i}$ is not restored, as seen from the argument in eq. (2.9).

Making use of eq. (2.9), we may now rewrite schematically the amplitude in eq. (2.1) as

$$
\mathcal{A}=\mathcal{H} \times \overline{\mathcal{S}} \times \prod_{i=1}^{2} J_{i}
$$

where the functions $\left\{J_{i}\right\}$ contain all relevant information associated with the collinear regions. Furthermore, in the remainder of this section, we will follow ref. [50] and define a 'non-collinear' factor

$$
H \equiv \mathcal{H} \times \overline{\mathcal{S}},
$$

where the reduced soft function is absorbed into the hard function. The factorized structure of the amplitude is then as shown in figure 1(a). Let us now describe how to generalise eq. (2.1) to NLP level, building on ref. [50]. First of all we wish to isolate the contributions to the radiative amplitude where the extra gluon is emitted by a collinearly enhanced configuration. With this in mind, and denoting the amplitude with an additional gluon emission by $\mathcal{A}_{\mu}$, one may naturally write

$$
\mathcal{A}_{\mu} \epsilon^{\mu}(k)=\mathcal{A}_{\mu}^{J} \epsilon^{\mu}(k)+\mathcal{A}_{\mu}^{H} \epsilon^{\mu}(k),
$$

where we are suppressing color indices, $\epsilon_{\mu}(k)$ is the polarization vector of the extra gluon, and $\mathcal{A}_{\mu}^{J}\left(\mathcal{A}_{\mu}^{H}\right)$ represent emissions from the jet (hard) functions, respectively. The amplitude for emission from collinear configurations can be defined as

$$
\mathcal{A}_{\mu}^{J}=\sum_{i=1}^{2} H\left(p_{i}-k ; p_{j}, n_{j}\right) J_{\mu}\left(p_{i}, k, n_{i}\right) \prod_{j \neq i} J\left(p_{j}, n_{j}\right) \equiv \sum_{i=1}^{2} \mathcal{A}_{\mu}^{J_{i}} .
$$




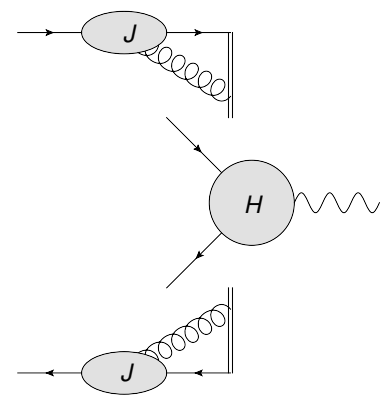

(a)

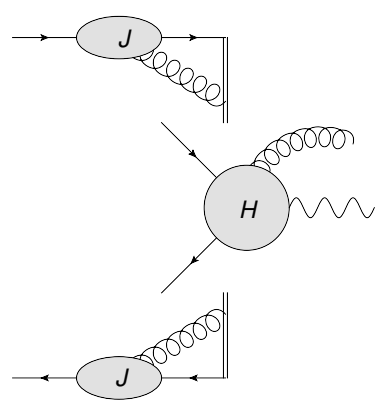

(b)

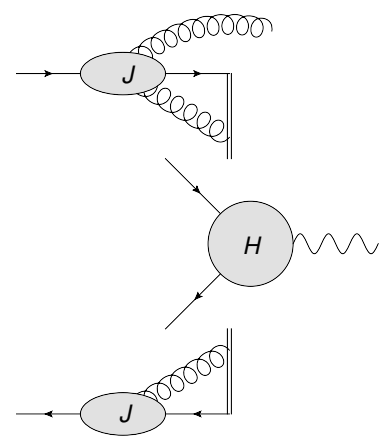

(c)

Figure 1. Schematic depiction of the factorization of the amplitude into the non-collinear function $H$ of eq. (2.11) and external jet functions: (a) portrays the non-radiative amplitude, while (b) and (c) contribute to the radiation of an extra gluon.

Here, for brevity, we have not displayed the dependence on the coupling and on $\epsilon$; we have introduced in $H$ the notation of separating with a semi-colon the 'active' momentum (here the momentum of the radiating leg) from the other vectors appearing in the function; most importantly, we have introduced the radiative jet function,

$$
J_{\mu}\left(p, n, k, \alpha_{s}\left(\mu^{2}\right), \epsilon\right) u(p)=\int d^{d} y \mathrm{e}^{-\mathrm{i}(p-k) \cdot y}\left\langle 0\left|\Phi_{n}(y, \infty) \psi(y) j_{\mu}(0)\right| p\right\rangle,
$$

representing the emission of a gluon from inside a jet function. It is useful to compare this definition with the non-radiative jet function defined in eq. (2.2). Here the incoming particle (a quark of momentum $p$ in this case) is absorbed by the field at position $y$, but, along the way, the insertion of the chromo-electric current $j_{\mu}$ causes the loss of momentum $k$ through the emission of a gluon. The gauge phase is then translated to infinite distance by the customary Wilson line. Finally the fact that fields are evaluated at different spacetime points requires a Fourier transform in order to get the momentum-space correlator. For the purposes of the present paper we can take for the current the QED-like expression

$$
j_{a}^{\mu}(x)=\bar{\psi}(x) \gamma^{\mu} T_{a} \psi(x),
$$

where $T^{a}$ is a color generator in the fundamental representation. In fact, we will continue to suppress color indices and matrices throughout the paper, except when especially relevant, since we will explicitly consider only QED-like diagrams and focus on contributions to the cross section proportional to $C_{F}^{2}$. Ultimately, however, we will need to generalize the definition in eq. (2.14) to the full non-abelian theory, and to gluon-originated jets as well. A natural generalization of eq. (2.15) is to consider the conserved (though not covariantly conserved) non-abelian current (see e.g. ref. [100])

$$
\mathfrak{j}_{a}^{\mu}(x)=f_{a}^{b c} F_{b}^{\mu \nu}(x) A_{\nu c}(x)+\bar{\psi}(x) \gamma^{\mu} T_{a} \psi(x),
$$

where $f_{a}^{b c}$ are $\mathrm{SU}(N)$ structure constants. This choice has all the physical characteristics required for our definition: for example it allows radiation from a gluon jet, and it includes 
the proper non-abelian corrections to gluon radiation from a quark jet. There are, however, significant differences between the two cases concerning the application of relevant Ward identities and the renormalization properties, which modify to some extent the reasoning given below in the 'abelian' case, without disrupting the general structure of the argument; therefore, we leave the full treatment of the non-abelian radiative jet to future work. We note that in either case eq. (2.14) defines a universal object, depending solely on the properties of the given external particle (including its spin), but not on the details of the specific hard interaction process. We will explore further how to calculate this object in section 3 ; here we begin by noting that the radiative jet function defined in eq. (2.14), with the current in eq. (2.15), obeys the simple Ward identity [50]

$$
k^{\mu} J_{\mu}\left(p, n, k, \alpha_{s}\left(\mu^{2}\right), \epsilon\right)=q J\left(p, n, \alpha_{s}\left(\mu^{2}\right), \epsilon\right),
$$

where $q= \pm 1$ according to whether the momentum $p$ is incoming or outgoing respectively ( $q$ would represent the electric charge in a QED calculation). We may similarly consider the Ward identity for the entire amplitude: in this case, it takes the form

$$
k^{\mu} \mathcal{A}_{\mu}=0
$$

which in turn implies

$$
k^{\mu} \mathcal{A}_{\mu}^{H}=-k^{\mu} \mathcal{A}_{\mu}^{J} .
$$

We can use this result to relate emissions from inside the hard function, collected in the function $\mathcal{A}_{\mu}^{H}$, to those from the jet functions, represented by $\mathcal{A}_{\mu}^{J}$. Indeed, note that so far $\mathcal{A}_{\mu}^{H}$ has been defined as a matching condition: the Ward identity shows that it is not independent from $\mathcal{A}_{\mu}^{J}$. To be explicit, we may use eqs. (2.13) and (2.17) to find that

$$
\begin{aligned}
k^{\mu} \mathcal{A}_{\mu}^{J}\left(p_{i}, k\right) & =\sum_{i=1}^{2} q_{i} H\left(p_{i}-k ; p_{j}, n_{j}\right) \prod_{j=1}^{2} J\left(p_{j}, n_{j}\right) \\
& =\sum_{i=1}^{2} q_{i}\left[H\left(p_{i} ; p_{j}, n_{j}\right)+k^{\mu}\left(\frac{\partial}{\partial k^{\mu}} H\left(p_{i}-k ; p_{j}, n_{j}\right)\right)_{k \rightarrow 0}\right] \prod_{j} J\left(p_{j}, n_{j}\right),
\end{aligned}
$$

where we have Taylor expanded up to next-to-soft order in $k$ in the second line. We may also rewrite the derivative in the second line as

$$
\left.\frac{\partial}{\partial k^{\mu}} H\left(p_{i}-k ; p_{j}, n_{j}\right)\right|_{k \rightarrow 0}=-\frac{\partial}{\partial p_{i}^{\mu}} H\left(p_{i} ; p_{j}, n_{j}\right) .
$$

The zeroth order term in eq. (2.20) now can be seen to vanish due to charge conservation (or rather color conservation in the QCD case), and the Ward identity of eq. (2.19) implies

$$
\mathcal{A}_{\mu}^{H}\left(p_{i}, k\right)=\sum_{i=1}^{2} q_{i}\left(\frac{\partial}{\partial p_{i}^{\mu}} H\left(p_{i} ; p_{j}, n_{j}\right)\right) \prod_{j=1}^{2} J\left(p_{j}, n_{j}\right) .
$$

The reader may wonder at this point whether it might be possible to add a separate transverse contribution to the right-hand side. Such a contribution is argued to be absent 
at the level of the amplitude in ref. [48] (see also [89, 90]), based on gauge invariance and locality considerations. Here we notice that the $n_{i}^{\mu}$ dependence of individual factors in eq. (2.22) may in principle allow for transverse $\mathcal{O}\left(k^{0}\right)$ contributions, for example of the form $k^{\mu} /\left(n_{i} \cdot k\right)$. All such contributions must however cancel in the complete amplitude, and we will not include them in our analysis.

Returning now to the amplitude for emission from the jet functions, it is convenient, following refs. [50, 101], to introduce, for each jet, a decomposition of the polarization $\operatorname{sum}^{5}$ as

$$
\eta^{\mu \nu}=G^{\mu \nu}+K^{\mu \nu}, \quad K^{\mu \nu}(p ; k)=\frac{(2 p-k)^{\nu}}{2 p \cdot k-k^{2}} k^{\mu},
$$

so that $G_{\mu \nu}$ satisfies

$$
p^{\mu} G_{\mu \nu}=\mathcal{O}(k), \quad G_{\mu \nu} k^{\nu}=0 .
$$

This decomposition leads to a particularly simple expression for the emission of a $K$-gluon from the jets. Indeed, the Ward identity in eq. (2.17), together with eq. (2.13), yields

$$
\begin{aligned}
\mathcal{A}_{\nu}^{J_{i}} K_{i}^{\nu \mu} & =q_{i} \frac{\left(2 p_{i}-k\right)^{\mu}}{2 p_{i} \cdot k-k^{2}} H\left(p_{i}-k ; p_{j}, n_{j}\right) \prod_{j=1}^{2} J\left(p_{j}, n_{j}\right) \\
& =q_{i}\left[\frac{\left(2 p_{i}-k\right)^{\mu}}{2 p_{i} \cdot k-k^{2}} \mathcal{A}-\left(K_{i}^{\nu \mu} \frac{\partial}{\partial p_{i}^{\nu}} H\left(p_{i} ; p_{j}, n_{j}\right)\right) \prod_{j=1}^{2} J\left(p_{j}, n_{j}\right)\right],
\end{aligned}
$$

where $K_{i}^{\nu \mu}$ is the $K$ tensor appropriate to the $i$-th jet, we have again Taylor expanded in $k$, and we have recognized the non-radiative amplitude $\mathcal{A}$ in the second line. Upon combining this result with the emission from the hard function, as given in eq. (2.22), one obtains

$$
\mathcal{A}^{H, \mu}+\sum_{i=1}^{2} \mathcal{A}_{\nu}^{J_{i}} K_{i}^{\nu \mu}=\sum_{i=1}^{2} q_{i}\left[\frac{\left(2 p_{i}-k\right)^{\mu}}{2 p_{i} \cdot k-k^{2}} \mathcal{A}+\left(G_{i}^{\nu \mu} \frac{\partial}{\partial p_{i}^{\nu}} H\left(p_{i} ; p_{j}, n_{j}\right)\right) \prod_{j} J\left(p_{j}, n_{j}\right)\right] \text {. }
$$

Using eq. (1.2), one may rewrite this in a more useful form, as

$$
\begin{aligned}
\mathcal{A}^{H, \mu}+\sum_{i=1}^{2} \mathcal{A}_{\nu}^{J_{i}} K_{i}^{\nu \mu}= & \sum_{i=1}^{2} q_{i}\left[\left(\frac{\left(2 p_{i}-k\right)^{\mu}}{2 p_{i} \cdot k-k^{2}}+G_{i}^{\nu \mu} \frac{\partial}{\partial p_{i}^{\nu}}\right) \mathcal{A}\right. \\
& \left.-H\left(p_{i} ; p_{j}, n_{j}\right) G_{i}^{\nu \mu} \frac{\partial}{\partial p_{i}^{\nu}} \prod_{j=1}^{2} J\left(p_{j}, n_{j}\right)\right],
\end{aligned}
$$

so that derivatives with respect to hard momenta act only on the full non-radiative amplitude, or on the process-independent jet functions.

We must now, finally, consider the emission of a $G$-gluon from the jets, which is given by

$$
\sum_{i=1}^{2} \mathcal{A}_{\nu}^{J_{i}} G_{i}^{\nu \mu}=\sum_{i=1}^{2} G_{i}^{\nu \mu} H\left(p_{i}-k ; p_{j}, n_{j}\right) J_{\nu}\left(p_{i}, k, n_{i}\right) \prod_{j \neq i} J\left(p_{j}, n_{j}\right) .
$$

\footnotetext{
${ }^{5}$ Our definition of the $G$ and $K$ tensors differs from that of ref. [50] due to the fact that we have taken the momentum $k$ to be outgoing. Note also that in our calculation $k^{2}=0$, although most of the argument goes through also for off-shell $k_{\mu}$.
} 
As argued in ref. [50], projection with the $G$ tensor ensures that this contribution starts at next-to-leading power in the soft expansion. One may therefore Taylor expand the shifted hard function, keeping only the zeroth order term. Adding this to eq. (2.27), the radiative amplitude finally becomes

$$
\begin{aligned}
\mathcal{A}^{\mu}\left(p_{j}, k\right)= & \sum_{i=1}^{2}\left[q_{i}\left(\frac{\left(2 p_{i}-k\right)^{\mu}}{2 p_{i} \cdot k-k^{2}}+G_{i}^{\nu \mu} \frac{\partial}{\partial p_{i}^{\nu}}\right) \mathcal{A}\left(p_{i} ; p_{j}\right)\right. \\
& \left.+\mathcal{H}\left(p_{j}, n_{j}\right) \overline{\mathcal{S}}\left(\beta_{j}, n_{j}\right) G_{i}^{\nu \mu}\left(J_{\nu}\left(p_{i}, k, n_{i}\right)-q_{i} \frac{\partial}{\partial p_{i}^{\nu}} J\left(p_{i}, n_{i}\right)\right) \prod_{j \neq i} J\left(p_{j}, n_{j}\right)\right],
\end{aligned}
$$

where we have restored the factorized expression for the non-collinear function $H$ in terms of the hard factor $\mathcal{H}$ and the reduced soft factor $\overline{\mathcal{S}}$.

In the remainder of the paper, we will provide strong evidence that eq. (2.29), first derived with slight modifications in ref. [50], provides the missing ingredient for a complete reconstruction of NLP threshold logarithms in electroweak annihilation cross sections, generalizing the classic leading-power factorization formula given in eq. (1.2). Some comments are in order.

- We expect, and we will confirm below, that when eq. (2.29) is used to construct a NNLO annihilation cross section, by contracting it with the corresponding treelevel matrix element and integrating over phase space, it will correctly generate all threshold logarithms up to NLP. Phase space integration does not present difficulties, since next-to-soft contributions to eq. (2.29) can be integrated with the well-known factorized expression for the phase-space measure at leading power, while correction to phase space are only needed for leading-power matrix elements, and were discussed in detail in ref. [57].

- A new universal quantity arises at this level, the radiative jet function, defined originally in ref. [50]. In order to predict NLP logarithms in specific scattering processes, one must calculate this quantity, which is the subject of the following section.

- The only process-dependent contributions on the right-hand side of eq. (2.29) are the full non-radiative amplitude $\mathcal{A}$ and the hard function $\mathcal{H}$.

- At the level of singular contributions, our result complements the analysis of ref. [50] by correctly accounting for the soft and soft-collinear regimes, and in particular by subtracting the double counting of the latter with the inclusion of eikonal jet functions.

- It is important to note that our treatment suffices for electroweak annihilation cross sections, which do not involve final-state QCD jets, since all radiated gluons must be (next-to-) soft in this case. In the presence of final-state hadrons (for example in the case of Deep Inelastic Scattering (DIS), NLP threshold logarithms may in principle be associated with hard collinear emission. These are potentially not taken into account in eq. (2.29), which relies upon the soft expansion. 
Having now presented our general framework, we turn to the calculation of the radiative jet function defined by eq. (2.14), focusing in the present case on the abelian-like contributions generated by the current in eq. (2.15), up to one loop.

\section{The radiative jet function}

Eq. (2.29) expresses the radiative amplitude for electroweak annihilation in terms of a process-dependent hard function, together with a number of universal quantities. The soft function, the non-radiative jet and its eikonal counterpart are already defined at leading power in the soft expansion, and can in principle be taken over without changes. One also encounters, however, a new universal spin-dependent quantity: the radiative jet function of eq. (2.14). This quantity is defined for finite emitted energy $k$, and must be computed with NLP accuracy in the soft expansion, while keeping control of all collinear singularities which might interfere with the soft emission: all these contributions are expected to give rise to threshold logarithms when contracted with the tree-level complex-conjugate amplitude and integrated over the soft gluon phase space.

To substantiate our claims, in this section we will compute the radiative jet function to one loop order, selecting all terms that will contribute abelian-like threshold logarithms, proportional to $C_{F}^{2}$, to the NNLO cross-section. We will therefore choose the current in eq. (2.15), omit graphs containing three-gluon vertices, and pick the appropriate color structure for vertex corrections which would contribute terms proportional to $C_{A} C_{F}$, which we disregard. Before turning to that calculation, we will however pause to briefly discuss and motivate our choice of reference vectors $n^{\mu}$, which are necessary ingredients for nonradiative, eikonal and radiative jets.

\subsection{Jet functions for light-like $n^{\mu}$}

It is customary in the factorization literature to compute the jet functions appearing in eq. (1.2) with a reference vector off the light cone, $n^{2} \neq 0$ (see, for example, ref. [12]). This has several advantages, in particular in the case of all-order proofs and for renormalization group studies. Most notably, keeping $n^{\mu}$ off the light cone prevents the appearance of collinear divergences associated with the $n^{\mu}$ Wilson line, which are spurious and should in general be removed; furthermore, keeping $n$ generic allows to test perturbative calculations by verifying their independence on $n$; finally, space-like $n$ preserves certain analytic properties of the amplitude which are useful for all-order analyses. The non-radiative jet function $J$, and its eikonal counterpart $\mathcal{J}$, are well known and they have been computed at one loop for arbitrary (non-null) $n$ for example in refs. $[1,13]$.

Here we would like to argue that in the present case there are considerable computational advantages to be gained by keeping $n^{2}=0$, and at the same time the issues of interpretation, that can be quite subtle for generic correlators, can easily be dealt with for the radiative amplitude that we are discussing here. To illustrate the problem, let us begin by considering the one-loop calculation of the non-radiative jet function $J(p, n)$. For an external incoming quark, one must consider the diagrams in figure 2 , where the second graph denotes a UV counterterm associated with the renormalization of the vertex, 
and we have omitted the similar counterterm graphs associated with external leg corrections. For $n^{2} \neq 0$, the complete jet function must depend on the dimensionless variable $z \equiv(p \cdot n)^{2} /\left(n^{2} \mu^{2}\right)$, as a consequence of the invariance of the eikonal Feynman rules under the rescaling $n^{\mu} \rightarrow \kappa n^{\mu}$. Furthermore, the function is well defined in dimensional regularization due to the presence of the energy scale $p \cdot n$. For $n^{2}=0$, both of these properties are lost: dependence on $p \cdot n$ is in principle ruled out by rescaling invariance, so that all integrals arising in the relevant Feynman diagrams are effectively scale-less, and must be defined to vanish in dimensional regularization. In fact, there is an extra twist: while the diagrams vanish, one finds that there is a residual dependence on $p \cdot n$ in the UV counterterms, due to an anomalous breaking of rescaling invariance originating from the collinear pole associated with emission from the light-like Wilson line [13, 19].

To give a concrete example, consider the first diagram in figure 2, which we denote by $J_{\mathrm{V}}^{(1)}$. In $d=4-2 \epsilon$ dimensions, and for $n^{2}=0$, it is given by

$$
\begin{aligned}
J_{\mathrm{V}}^{(1)}(p, n ; \epsilon) & =2 \mathrm{i} \mu^{2 \epsilon} g_{s}^{2} \int \frac{d^{d} k}{(2 \pi)^{d}} \frac{(\not p-\not k) \not h}{k^{2} 2 n \cdot k(p-k)^{2}} \\
& =2 \mathrm{i} \mu^{2 \epsilon} g_{s}^{2} \int \frac{d^{d} k}{(2 \pi)^{d}} \int_{0}^{1} d x \int_{0}^{1} d y \frac{2 y(\not p-\not k) \not h}{\left[y k^{2}-2 x y k \cdot p+2(1-y) n \cdot k\right]^{3}} .
\end{aligned}
$$

In the second line, we have introduced Feynman parameters, and we note the characteristic parameter dependence of the $k^{2}$ term in the denominator, which arises in the presence of linear denominators. Carrying out the momentum integration and using the Dirac equation, one may rewrite this as

$$
J_{\mathrm{V}}^{(1)}(p, n ; \epsilon)=\frac{\alpha_{s}}{2 \pi}\left(4 \pi \mu^{2}\right)^{\epsilon} \Gamma(1+\epsilon)(-2 p \cdot n)^{-\epsilon} \frac{1}{\epsilon(\epsilon-1)} \int_{0}^{1} d y y^{-1+\epsilon}(1-y)^{-1-\epsilon} .
$$

At this point, one might be tempted to interpret directly the $y$ integral as $B(\epsilon,-\epsilon)=0$. More accurately, one observes that the integral is not well defined for any values of $\epsilon$, and must therefore be defined to vanish in dimensional regularization. In this simple case, it is actually easy to disentangle the ultraviolet divergence (arising from the region $y \rightarrow 0$ ) from the infrared one (arising from the region $y \rightarrow 1$ ). One may simply insert a factor of $(1-y)+y=1$ to see explicitly that infrared and ultraviolet poles cancel exactly; using different regulators yields compatible results. The standard treatment at this point is compute the renormalized jet function, isolating the ultraviolet divergence in eq. (3.2) and defining the appropriate $\overline{\mathrm{MS}}$ counterterm to subtract it. The sum of $J_{V}^{(1)}$ and $J_{V, C T}^{(1)}$ is then simply the negative of the UV pole of $J_{V}^{(1)}$, which is correctly interpreted as an infrared divergence. For $n^{2}=0$ one recovers the expected double soft-collinear pole because of the explicit collinear divergence in eq. (3.2). The external leg corrections in figure 2 can be treated similarly. In what follows, we will take the alternative route of computing bare jet functions, which will allow us to simplify considerably all calculations, and make a more direct contact with the calculation performed with the method of regions in ref. [64], and eventually with the SCET approach described in ref. [96]. This approach works for our current problem, and for the choice $n^{2}=0$, because of the simple renormalization 


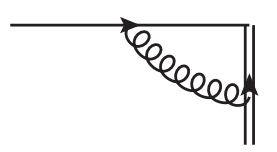

$J_{\mathrm{V}}^{(1)}$

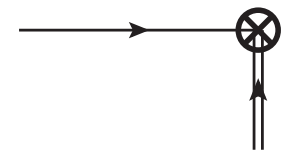

$J_{\bigvee, C T}^{(1)}$

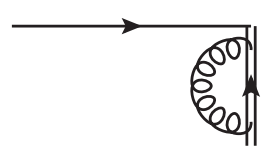

$J_{n^{2}}^{(1)}$

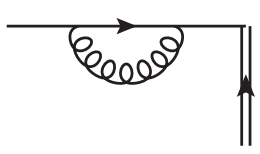

$J_{\mathrm{P}}^{(1)}$

Figure 2. Feynman diagrams contributing to the one-loop jet function. Here $J_{V, C T}^{(1)}$ denotes the counterterm associated with the vertex graph, $J_{V}^{(1)}$, while counterterms associated with external leg corrections have been omitted.

properties of eq. (2.29). More specifically, we note that the left-hand side of eq. (2.29) is an on-shell scattering amplitude, and thus it is not renormalized: all counterterms needed for the various factors must cancel. Furthermore, the right-hand side can be expressed almost entirely in terms of the complete non-radiative amplitude $\mathcal{A}$, which is also renormalizationgroup ( $\mathrm{RG}$ ) invariant. The first line of eq. (2.29) is already in this form; the second line can be brought to a similar form simply by noting that, in each term in the sum over external partons, only one non-radiative jet function is missing to reconstruct the full non-radiative amplitude. Multiplying and dividing by that jet one can write eq. (2.29) as

$$
\begin{aligned}
\mathcal{A}^{\mu}\left(p_{j}, k\right)= & \sum_{i=1}^{2}\left\{q_{i}\left(\frac{\left(2 p_{i}-k\right)^{\mu}}{2 p_{i} \cdot k-k^{2}}+G_{i}^{\nu \mu} \frac{\partial}{\partial p_{i}^{\nu}}\right)\right. \\
& \left.+G_{i}^{\nu \mu}\left[\frac{J_{\nu}\left(p_{i}, k, n_{i}\right)}{J\left(p_{i}, n_{i}\right)}-q_{i} \frac{\partial}{\partial p_{i}^{\nu}}\left(\ln J\left(p_{i}, n_{i}\right)\right)\right]\right\} \mathcal{A}\left(p_{i} ; p_{j}\right) .
\end{aligned}
$$

In this form, it is evident that the factor in square brackets in the second line must be RG invariant by itself, to all orders in perturbation theory. This is easily verified by inspection, noting from the diagrammatic expansion that the UV divergences of the radiative jet function $J_{\mu}$ are the same as those of the non-radiative jet $J$, simply multiplied by the treelevel expression for $J_{\mu}$. We are thus free to compute eq. (3.3) in either bare or renormalized perturbation theory. The advantage of using a light-like reference vector is now apparent: with $n^{2}=0$, radiative corrections to the bare non-radiative jet function vanish to all orders in perturbation theory, and one can simplify eq. (3.3) by setting

$$
J\left(p_{i}, n_{i}\right)=1 .
$$

The term containing the derivative of the non-radiative jet function now vanishes, and we are left with the simple expression ${ }^{6}$

$$
\mathcal{A}^{\mu}\left(p_{j}, k\right)=\sum_{i=1}^{2}\left(q_{i} \frac{\left(2 p_{i}-k\right)^{\mu}}{2 p_{i} \cdot k-k^{2}}+q_{i} G_{i}^{\nu \mu} \frac{\partial}{\partial p_{i}^{\nu}}+G_{i}^{\nu \mu} J_{\nu}\left(p_{i}, k\right)\right) \mathcal{A}\left(p_{i} ; p_{j}\right) .
$$

\footnotetext{
${ }^{6}$ Strictly speaking, one could write eq. (3.5) explicitly in terms of the ratio $\tilde{J}_{\mu}\left(p_{i}, k\right)=J_{\mu}\left(p_{i}, k\right) / J\left(p_{i}\right)$. As eq. (3.4) makes clear, $J_{\mu}\left(p_{i}, k\right)=\tilde{J}_{\mu}\left(p_{i}, k\right)$ in dimensional regularisation. As well as being UV finite, the ratio is also free of soft (but not collinear) singularities due to the correspondence between UV and IR poles. If a different regularisation scheme is used, these properties persist for $\tilde{J}_{\mu}$, but not for $J_{\mu}$ itself.
} 
We will use this explicit form in section 4, and we believe that the simple formal properties embodied in eq. (3.3) and eq. (3.5) will be useful also for the all-order analysis that will have to be performed in order to construct a resummation procedure for NLP threshold logarithms.

\subsection{The radiative jet function at one loop}

The main result of section 3.1 is that, for the purposes of the present calculation, we are allowed to work with bare quantities, and with light-like reference vectors for jets. This has considerably simplified our task, since perturbative corrections to non-radiative (eikonal) jet functions can be taken to vanish to all orders. The same is of course not true for the radiative jet function defined in eq. (2.14), which in fact depends on several momentum scales. We will now evaluate this function at one loop, using a light-like reference vector, and working in bare perturbation theory as required by our reasoning in section 3.1.

We begin by defining the perturbative coefficients of the radiative jet function via $^{7}$

$$
J_{\nu}\left(p, n, k ; \alpha_{s}, \epsilon\right)=g_{s} \sum_{n=0}^{\infty}\left(\frac{\alpha_{s}}{4 \pi}\right)^{n} J_{\nu}^{(n)}(p, n, k ; \epsilon) .
$$

The operator definition in eq. (2.14) gives a straightforward result at tree level, consisting of a single emission from the external leg of momentum $p$, and yielding the expression

$$
\begin{aligned}
J^{\nu(0)}(p, n, k) & =\frac{\not k \gamma^{\nu}}{2 p \cdot k}-\frac{p^{\nu}}{p \cdot k} \\
& =-\frac{p^{\nu}}{p \cdot k}+\frac{k^{\nu}}{2 p \cdot k}-\frac{\mathrm{i} k_{\alpha} \Sigma^{\alpha \mu}}{2 p \cdot k} .
\end{aligned}
$$

In the second line, we have chosen to decompose the result into spin-independent and spindependent parts, where the latter takes the form of a magnetic moment coupling to the fermion leg, involving the Lorentz generators

$$
\Sigma^{\alpha \nu}=\frac{\mathrm{i}}{4}\left[\gamma^{\alpha}, \gamma^{\nu}\right]
$$

The expression in the second line of eq. (3.7) naturally generalizes to the case of emission from hard partons of different spin, simply by choosing the appropriate form for the corresponding Lorentz generator.

At one loop, things are more complicated, and the relevant contributions are depicted in figure 3. Diagrams (a) and (b) have a more intricate kinematic dependence, as they link the physical external leg to the Wilson line, while diagrams (c) and (d) are standard radiative corrections to the emission amplitude. External leg corrections are not included since they are pure counterterms. The diagrams linking the parton line and the Wilson line are readily evaluated. For our purposes, we need to include terms up to $\mathcal{O}(\epsilon)$, since phasespace integration over $k$ will generate singularities. On the other hand, we can neglect

\footnotetext{
${ }^{7}$ Given that we are explicitly talking about QCD corrections from now on, we have replaced the electromagnetic charge factor $q_{i}$ in section 2 with the strong coupling $g_{s}$. Note that in our convention the sign of $q_{i}$ is negative for an incoming quark line.
} 


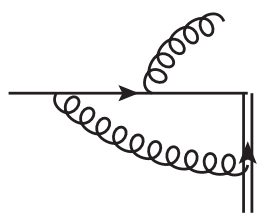

$J_{\text {ą }}^{\nu(1)}$

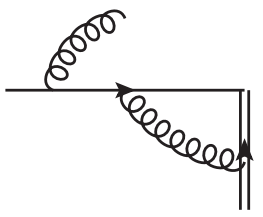

$J_{\mathrm{b}}^{\nu(1)}$

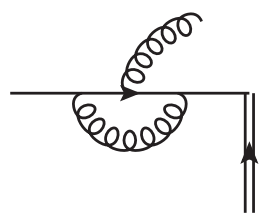

$J_{c}^{\nu(1)}$

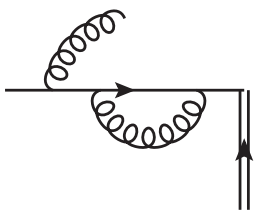

$J_{\mathrm{d}}^{\nu(1)}$

Figure 3. Contributions to the QED-like terms in the one-loop bare radiative jet function.

terms quadratic in $k^{\mu}$ since they cannot contribute to NLP threshold logarithms. The result is

$$
\begin{aligned}
J_{a+b}^{\nu(1)}(p, n, k ; \epsilon)= & (2 p \cdot k)^{-\epsilon}\left\{\left(\frac{2}{\epsilon}+4+8 \epsilon\right)\left[\frac{n \cdot k}{p \cdot k} \frac{p^{\nu}}{p \cdot n}-\frac{\not k \gamma^{\nu}}{2 p \cdot k}-\frac{n^{\nu}}{p \cdot n}\right]\right. \\
& \left.+(1+3 \epsilon)\left(-\frac{2 k^{\nu}}{p \cdot k}+\frac{\gamma^{\nu} \not h}{p \cdot n}-\frac{p^{\nu} \not k \not h}{p \cdot k p \cdot n}\right)\right\} .
\end{aligned}
$$

Notice that this combination has only a single pole, while the individual diagrams (a) and (b) have (canceling) double poles. Diagrams (c) and (d) do not depend on the reference vector $n$, and they yield

$$
\begin{aligned}
J_{c+d}^{\nu(1)}(p, n, k ; \epsilon)= & (2 p \cdot k)^{-\epsilon}\left[\frac{1}{\epsilon}\left(\frac{\not k \gamma^{\nu}}{p \cdot k}+\frac{k^{\nu}}{p \cdot k}\right)+\frac{5}{2} \frac{\not k \gamma^{\nu}}{p \cdot k}+\frac{k^{\nu}}{p \cdot k}\right. \\
& \left.+\epsilon\left(5 \frac{\not k \gamma^{\nu}}{p \cdot k}+2 \frac{k^{\nu}}{p \cdot k}\right)\right] .
\end{aligned}
$$

The full result for the bare one-loop radiative jet function is then given by

$$
\begin{aligned}
J^{\nu(1)}(p, n, k ; \epsilon)= & (2 p \cdot k)^{-\epsilon}\left[\left(\frac{2}{\epsilon}+4+8 \epsilon\right)\left(\frac{n \cdot k}{p \cdot k} \frac{p^{\nu}}{p \cdot n}-\frac{n^{\nu}}{p \cdot n}\right)-(1+2 \epsilon) \frac{\mathrm{i} k_{\alpha} \Sigma^{\alpha \nu}}{p \cdot k}\right. \\
& \left.+\left(\frac{1}{\epsilon}-\frac{1}{2}-3 \epsilon\right) \frac{k^{\nu}}{p \cdot k}+(1+3 \epsilon)\left(\frac{\gamma^{\nu} \not h}{p \cdot n}-\frac{p^{\nu} \not k \not h}{p \cdot k p \cdot n}\right)\right]+\mathcal{O}\left(\epsilon^{2}, k\right),
\end{aligned}
$$

where we have again chosen to write this in terms of spin-independent and spin-dependent components, for the $n$-independent terms. A comment is in order regarding the $\epsilon$ pole appearing in this result. As explained in the previous section, we have avoided the introduction of UV counterterms given that these will cancel when all ingredients in eq. (3.5) are combined. It follows that the singularity appearing here can be interpreted directly as the infrared pole that will appear in the final amplitude.

An important check of the above results is the Ward identity, eq. (2.17). We note first that it is verified by the tree-level jet emission function in eq. (3.7): indeed

$$
k_{\nu} J^{\nu(0)}(p, n, k)=-g_{s} J^{(0)}(p, n)
$$


where we have used the fact that, at tree-level, $J^{(0)}(p, n)=1$, and that our expression has been derived for $k^{2}=0$, as well as our convention for the sign of the charge of an incoming fermion. At the one-loop level, the results of eqs. (3.11) and 3.12) imply that

$$
k_{\nu} J^{\nu(1)}=0=-g_{s} J^{(1)}
$$

given that loop corrections to the non-radiative jet function vanish. Note again that we are verifying the Ward identity only up to corrections $\sim \mathcal{O}\left(k^{2}\right)$, since our jet functions have been computed for on-shell $k$.

In eq. (3.3), one must finally contract the jet emission function with the $G$ tensor defined in eq. (2.23). The reader may verify that, remarkably, the one-loop expression in eq. (3.11) is an eigenstate of $G$. More precisely one finds

$$
G^{\nu \mu} J_{\nu}^{(1)}(p, n, k)=J_{\nu}^{(1)}(p, n, k) .
$$

We now have everything we need in order to test our factorized expression for the radiative amplitude, eq. (3.5), in the context of a NNLO calculation. To this end, we consider Drell-Yan production in the following section.

\section{Application to Drell-Yan production}

In the previous sections, we have assembled the ingredients needed to organize threshold corrections at next-to-leading power, at the level of scattering amplitudes. As already mentioned above, we will now test our results in the concrete example of Drell-Yan production at NNLO, with the goal of reproducing abelian-like logarithms (weighted by the color factor $C_{F}^{2}$ ) in graphs involving the real radiation of a (next-to-) soft gluon, dressed by a virtual correction. This is a non-trivial check that our formalism is correct, given that one expects an interplay between soft and collinear contributions when virtual gluons are included.

Drell-Yan production is the simplest testing ground for our formalism, since it involves only two colored particles at leading order, it has a non-trivial abelian-like limit, and it does not involve final state jets; furthermore, NNLO corrections have been known for many years $[45,65,102,103]$. A more interesting process for phenomenological applications is Higgs production via gluon fusion, where NLP logarithms are now known at $\mathrm{N}^{3} \mathrm{LO}$ [73, 74] (see also refs. [104, 105]): we plan to tackle that process in future work, when the full non-abelian generalization of our formalism has been worked out.

We recall that, for the purely real emission contributions, involving two real gluons at NNLO, all threshold logarithms at both LP and NLP have been shown to arise from the soft expansion, with no contamination from collinear singularities, as has been verified up to NNLO in ref. [57]. Mixed real-virtual corrections at NNLO, which we examine here, are the first instance in which the collinear generalization of Low's theorem is required. In a diagrammatic framework, the various contributions involved have been analysed recently in ref. [64], using the method of regions of refs. [70-72]. We will make contact with this analysis in what follows. 


\subsection{The real-virtual Drell-Yan K-factor at NNLO}

We begin by very briefly setting up or notations and conventions. The leading-order DrellYan process for the production of an off-shell vector boson $V^{*}$ of invariant mass $Q^{2}$ proceeds through the process

$$
q(p)+\bar{q}(\bar{p}) \rightarrow V^{*}(Q),
$$

where $q$ and $\bar{q}$ denote a quark and antiquark respectively, and arguments label their fourmomenta. Defining the squared centre-of-mass energy $s=(p+\bar{p})^{2}$, one may introduce the dimensionless threshold variable

$$
z=\frac{Q^{2}}{s}
$$

representing the fraction of available energy carried by the final state photon; the threshold limit then corresponds to $z \rightarrow 1$. The K-factor at fixed order in perturbation theory is defined by

$$
K^{(n)}(z)=\frac{1}{\sigma^{(0)}} \frac{d \sigma^{(n)}(z)}{d z},
$$

where $\sigma^{(n)}$ is the $n$-loop Drell-Yan cross section. For the case of emission of a single real gluon with momentum $k$, one may introduce the Mandelstam invariants

$$
t=-2 k \cdot p, \quad u=-2 k \cdot \bar{p},
$$

which may be parametrized as [68]

$$
t=-2 s(1-y)(1-z), \quad u=-2 s y(1-z),
$$

where $0<y<1$. Using these variables, and setting, for simplicity, the renormalization scale $\mu^{2}=Q^{2}$, the real-virtual contribution to the NNLO K-factor can be written as

$$
K_{\mathrm{rv}}^{(2)}(z)=\frac{1}{16 \pi^{2}} \frac{(4 \pi)^{\epsilon}}{\Gamma(2-\epsilon)} z^{\epsilon}(1-z)^{1-2 \epsilon} \int_{0}^{1} d y[y(1-y)]^{-\epsilon}\left[\mathcal{A}_{\mathrm{rv}}^{\dagger} \mathcal{A}_{\mathrm{r}}+\mathcal{A}_{\mathrm{r}}^{\dagger} \mathcal{A}_{\mathrm{rv}}\right],
$$

where $\mathcal{A}_{\mathrm{rv}}$ and $\mathcal{A}_{\mathrm{r}}$ are the amplitudes for single real gluon emission, at NLO and LO respectively.

Although the complete NNLO Drell-Yan K-factor has been known for a long time [68], no separate result exists in the literature for the real-virtual contribution. We have reproduced the relevant calculation, and we give here the result ${ }^{8}$ for threshold contributions up to NLP. One finds

$$
\begin{aligned}
K_{\mathrm{rv}}^{(2)}(z)=\left(\frac{\alpha_{s}}{4 \pi} C_{F}\right)^{2}\{ & \frac{32}{\epsilon^{3}}\left[\mathcal{D}_{0}(z)-1\right]+\frac{16}{\epsilon^{2}}\left[-4 \mathcal{D}_{1}(z)+3 \mathcal{D}_{0}(z)+4 L(z)-6\right] \\
& +\frac{4}{\epsilon}\left[16 \mathcal{D}_{2}(z)-24 \mathcal{D}_{1}(z)+32 \mathcal{D}_{0}(z)-16 L^{2}(z)+52 L(z)-49\right] \\
& -\frac{128}{3} \mathcal{D}_{3}(z)+96 \mathcal{D}_{2}(z)-256 \mathcal{D}_{1}(z)+256 \mathcal{D}_{0}(z) \\
& \left.+\frac{128}{3} L^{3}(z)-232 L^{2}(z)+412 L(z)-408\right\}
\end{aligned}
$$

\footnotetext{
${ }^{8}$ Here and throughout, for brevity, we neglect terms involving transcendental constants, as was done in refs. [57, 64]. Such terms do not contain any new information.
} 
where we defined

$$
\mathcal{D}_{n}(z)=\left(\frac{\log ^{n}(1-z)}{(1-z)}\right)_{+}, \quad L(z)=\log (1-z) .
$$

Further details of the calculation can be found in appendix A. Note that we do not include the Dirac $\delta$-function contributions in eq. (4.7), as these mix with virtual corrections that are irrelevant for the purposes of this paper.

\subsection{Reconstructing the K-factor at NLP}

We are now going to reproduce eq. (4.7), starting from our factorized expression for the amplitude, given in eq. (3.5), and including the required contributions from the phase space measure at NLP in the threshold expansion. Since the calculation is not technically difficult, we simply outline the main steps and give the results for the various contributions to the K-factor, distinguishing their physical origin.

\subsubsection{NLP corrections to the phase-space measure}

Before discussing how the $\mathcal{D}_{n}(z)$ and $L^{n}(z)$ terms in (4.7) are reproduced in our factorized approach, we address the issue of how the amplitude factorization presented in eq. (2.29) and eq. (3.5) can be employed to obtain cross section results. ${ }^{9}$ Eq. (4.6) expresses the required contribution to the $\mathrm{K}$-factor in terms of the density

$$
\begin{aligned}
\mathcal{P} & =\mathcal{A}_{\mathrm{rv}}^{\dagger} \mathcal{A}_{\mathrm{r}}+\mathcal{A}_{\mathrm{r}}^{\dagger} \mathcal{A}_{\mathrm{rv}} \\
& =\mathcal{P}_{\mathrm{LP}}+\mathcal{P}_{\mathrm{NLP}}+\ldots,
\end{aligned}
$$

where the second line denotes the expansion in powers of $1-z$ up to NLP. This must be combined with the overall phase-space prefactor

$$
z^{\epsilon}(1-z)^{1-2 \epsilon}=(1-z)^{1-2 \epsilon}\left[1+\epsilon(1-z)+\mathcal{O}\left((1-z)^{2}\right)\right]
$$

which arises from the real gluon phase space.

Working at next-to-leading power in $(1-z)$ has the advantage that corrections to the phase space measure and to the matrix element do not interfere. One may, in fact, write the differential cross section schematically as

$$
d \sigma=d \Phi_{3, \mathrm{LP}}\left(\mathcal{P}_{\mathrm{LP}}+\mathcal{P}_{\mathrm{NLP}}\right)+d \Phi_{3, \mathrm{NLP}} \mathcal{P}_{\mathrm{LP}},
$$

where $d \Phi_{3,(\mathrm{~N}) \mathrm{LP}}$ denotes the three-particle phase space at the required order in the threshold expansion. In the present context, we see that we can proceed by integrating the full squared matrix element, given by eq. (3.5) contracted with the tree-level amplitude, with the eikonal expression for the phase-space measure; we can then include phase-space corrections, where however only the leading-power squared matrix element is needed. Note that

\footnotetext{
${ }^{9}$ Note that the present situation differs from that of ref. [73], where the three-loop results for the form factors contributing to Higgs and Drell-Yan production could be used to infer the coefficients of the distributions $\mathcal{D}_{n}(z)$ at $\mathrm{N}^{3} \mathrm{LO}$ for the corresponding cross sections; in fact, that procedure does not extend to the $L^{n}(z)$ terms.
} 
NLP corrections to the phase space measure affect the cross section to all orders, starting with the tree-level emission of a single gluon, as was already noted in ref. [57] in order to reproduce the known NLP logarithms in the one-loop Drell-Yan K-factor. Appendix B of that paper also included a discussion of how to obtain the NE contribution to a general multi-gluon phase space. In what follows, given the simplicity of the one-gluon-emission calculation, we will not make explicit use of eq. (4.11), which however would be useful in order to construct all-order expressions.

\subsubsection{From tree level to one loop}

In order to reproduce all NLP terms in the real-virtual contribution to the NNLO DrellYan K-factor, we must calculate all contributions appearing in eq. (3.3), before integrating over the real-gluon phase space according to eq. (4.6). Before performing this calculation at one-loop level, it is worth pausing to briefly remark upon what happens at tree level. In that case, the derivatives in eq. (3.3) and (3.5) act on momentum-independent leadingorder quantities, ${ }^{10}$ and thus vanish. The factor including the radiative jet function is easily computed using eq. (2.23) and eq. (3.7), and it gives

$$
G^{\nu \mu} J_{\nu}^{(0)}(p, n, k)=-\frac{\mathrm{i} k_{\alpha} \Sigma^{\alpha \mu}}{p \cdot k_{2}} J^{(0)}(p, n),
$$

where we have used the fact that $J^{(0)}(p, n)=1$. Using the leading-power factorization formula in eq. (1.2), one then writes

$$
\mathcal{H}\left(p_{i}, n_{i}\right) \overline{\mathcal{S}}\left(p_{i}, n_{i}\right) G_{i}^{\nu \mu} J_{\nu}\left(p_{i}, n_{i}, k\right) \prod_{j \neq i} J\left(p_{j}, n_{j}\right)=-\frac{\mathrm{i} k_{\alpha} \Sigma^{\alpha \mu}}{p_{i} \cdot k} \mathcal{A}\left(p_{i}, p_{j}\right) .
$$

This combines with the first term on the right-hand side of eq. (3.5) to reproduce the non-radiative amplitude dressed by a complete (spin-dependent) gluon emission. Upon integrating over the real gluon phase space, this precisely reproduces the NLO K-factor calculation, already carried out using an effective approach in ref. [57].

Returning now to the one-loop example, it is useful to distinguish three contributions to the cross section which are physically distinct. Let us begin by rewriting the one-loop contribution to eq. (3.5) as

$$
\begin{aligned}
\mathcal{A}^{\mu,(1)}\left(p_{j}, k\right)= & \sum_{i=1}^{2}\left[\left(\frac{\left(2 p_{i}-k\right)^{\mu}}{2 p_{i} \cdot k-k^{2}}+G_{i}^{\nu \mu} \frac{\partial}{\partial p_{i}^{\nu}}+G_{i}^{\nu \mu} J_{\nu}^{(0)}\left(p_{i}, k\right)\right) \mathcal{A}^{(1)}\left(p_{i} ; p_{j}\right)\right. \\
& \left.+G_{i}^{\nu \mu} J_{\nu}^{(1)}\left(p_{i}, k\right) \mathcal{A}^{(0)}\left(p_{i} ; p_{j}\right)\right]
\end{aligned}
$$

where we expanded the amplitudes in powers of $\alpha_{s} /(4 \pi)$, as in eq. (3.6). Using eq. (4.12), one finds that the first and third terms on the right-hand side combine to give the nonradiative amplitude, dressed by a complete (spin-dependent) gluon emission. This is directly analogous to the tree-level behaviour described above. Together with the remaining

\footnotetext{
${ }^{10}$ Note that the derivatives do not act on the momentum dependence implicit in the particle wave functions, for example the quark spinors in this case.
} 
terms, there are then three contributions to calculate: the dressed non-radiative amplitude, the derivative of the non-radiative amplitude, and the radiative jet contribution. We compute them in turn.

\subsubsection{The dressed non-radiative amplitude}

The one-loop non-radiative amplitude can be easily obtained from the well-known result for the one-loop quark form factor (see, for example, ref. [13]). Setting $\mu^{2}=Q^{2}$ and reabsorbing the factors of $\ln (4 \pi)$ and $\gamma_{E}$ in the renormalization scale, as appropriate to the $\overline{\mathrm{MS}}$ scheme, it is given by

$$
\mathcal{A}^{(1)}(z)=-\frac{\alpha_{s}}{4 \pi} z^{\epsilon} \frac{\Gamma^{2}(1-\epsilon) \Gamma(1+\epsilon)}{\Gamma(1-2 \epsilon)}\left(\frac{2}{\epsilon^{2}}+\frac{3}{\epsilon}+8+16 \epsilon+\mathcal{O}\left(\epsilon^{2}\right)\right),
$$

where the factor $z^{\epsilon}$ arises since the non-radiative amplitude must be computed with $(p+\bar{p})^{2}=s$. As is implicit in the above discussion, this dresses the tree-level amplitude involving one real gluon emission. The relevant amplitudes were reviewed recently in ref. [57]. The results for the squared matrix elements at leading and next-to-leading power are

$$
\left|\mathcal{A}_{\mathrm{r}, \mathrm{LP}}\right|^{2}=16(1-\epsilon) g_{s}^{2} \frac{s^{2}}{u t}
$$

and

$$
\mathcal{A}_{\mathrm{r}, \mathrm{NLP}}^{\dagger} \mathcal{A}_{\mathrm{r}, \mathrm{LP}}+\mathcal{A}_{\mathrm{r}, \mathrm{LP}}^{\dagger} \mathcal{A}_{\mathrm{r}, \mathrm{NLP}}=8(1-\epsilon) g_{s}^{2}\left(\frac{s}{t}+\frac{s}{u}\right)
$$

respectively, where again LP and NLP refer to the order in the expansion in powers of $(1-z)$. To obtain the appropriate contribution to the Drell-Yan K factor at NNLO, one must now multiply the sum of eqs. (4.16), (4.17) by the form factor contribution in eq. (4.15). After carrying out the phase space integration for the emitted gluon according to eq. (4.6), the result is

$$
\begin{aligned}
K_{\mathrm{dB}}^{(2)}(z)=\left(\frac{\alpha_{s}}{4 \pi} C_{F}\right)^{2}\{ & \frac{32}{\epsilon^{3}}\left[\mathcal{D}_{0}(z)-1\right]+\frac{8}{\epsilon^{2}}\left[-8 \mathcal{D}_{1}(z)+6 \mathcal{D}_{0}(z)+8 L(z)-14\right] \\
& +\frac{16}{\epsilon}\left[4 \mathcal{D}_{2}(z)-6 \mathcal{D}_{1}(z)+8 \mathcal{D}_{0}(z)-4 L^{2}(z)+14 L(z)-14\right] \\
& -\frac{128}{3} \mathcal{D}_{3}(z)+96 \mathcal{D}_{2}(z)-256 \mathcal{D}_{1}(z)+256 \mathcal{D}_{0}(z) \\
& \left.+\frac{128}{3} L^{3}(z)-224 L^{2}(z)+448 L(z)-512\right\} .
\end{aligned}
$$

Not surprisingly, all plus distributions are correctly reproduced by the dressed Born amplitude: the derivative and radiative jet contributions are expected to be strictly NLP, as we verify below.

\subsubsection{The derivative of the non-radiative amplitude}

The second term on the right-hand side of eq. (3.5) consists of the non-radiative amplitude, differentiated in turn with respect to each external momentum. Considering for example 
the derivative with respect to $p$, eq. (4.15) gives

$$
G^{\nu \mu}(p, k) \frac{\partial \mathcal{A}^{(1)}}{\partial p^{\nu}}=\left[-\frac{\epsilon}{p \cdot \bar{p}}\left(-p^{\mu}+\frac{\bar{p} \cdot k}{p \cdot k} \bar{p}^{\mu}\right)\right] \mathcal{A}^{(1)} .
$$

This derivative, and the corresponding one with respect to $\bar{p}$, must be contracted with the tree-level real-emission amplitude, before being integrated over phase space as in eq. (4.6). Including also complex conjugate graphs, one finds that the contribution to the $\mathrm{K}$ factor is

$$
K_{\partial \mathcal{A}}^{(2)}(z)=\left(\frac{\alpha_{s}}{4 \pi} C_{F}\right)^{2}\left\{\frac{32}{\epsilon^{2}}+\frac{16}{\epsilon}[-4 L(z)+3]+64 L^{2}(z)-96 L(z)+128\right\},
$$

which contributes at NLP, as expected.

\subsubsection{The radiative jet contribution}

The last part of the NLP K-factor comes from the second line of eq. (2.29), and is due to those radiative jet contributions that have not yet been included in the dressed nonradiative amplitude. These can be extracted from eq. (3.11). At this stage, it becomes inevitable to make a precise choice for the factorization vectors $n_{i}^{\mu}$ in the radiative jet functions. In the leading-power factorization formula, eq. (1.2), it is possible to engineer a complete cancellation of the $n$ dependence of the non-radiative jets: $n$-dependent poles cancel between jets and eikonal jets, while $n$-dependent non-singular terms are cancelled by the hard function $\mathcal{H}$. At NLP, we have already made a special choice by setting $n_{i}^{2}=0$; with this choice the presence of $n$-dependent poles in eq. (3.11) suggests that in order to achieve formal independence from $n$ we would need to introduce a further subtraction, replacing collinear poles in the direction $n_{i}$ with those associated with the physical momentum of the parton colliding with parton $i$. There is however a simple shortcut, already introduced in eq. (2.7): we may simply choose

$$
n=\bar{p}, \quad \bar{n}=p .
$$

This is physically motivated by the fact that the Wilson line in the direction $n_{i}$ acts as a replacement for the parton colliding with parton $i$; furthermore, considering for example emission from the parton with momentum $p$, one observes that $\bar{p}$ is the only other light-like vector in the problem; finally, the method-of-regions calculation of ref. [64] shows that this choice of reference vectors does indeed account for all the poles in the radiative amplitude. For processes with more legs, one would then choose $n_{i}$ to be the anti-collinear vector appearing in the Sudakov decomposition related to leg $i$, i.e. satisfying eq. (2.5), with $Q$ the appropriate hard scale.

With the choice in eq. (4.21), we proceed by contracting eq. (3.11) (for both the $p$ and $\bar{p}$ legs) with the NLO tree-level amplitude, including complex conjugate diagrams, and then we integrate over phase space. We find

$$
K_{\text {collinear }}^{(2)}(z)=\left(\frac{\alpha_{s}}{4 \pi} C_{F}\right)^{2}\left\{-\frac{16}{\epsilon^{2}}+\frac{4}{\epsilon}[12 L(z)-5]-72 L^{2}(z)+60 L(z)-24\right\},
$$

where the label 'collinear' for this contribution will be discussed in the following section. Once again, as expected, this contribution is strictly NLP. 


\subsection{Discussion}

We have now calculated all necessary ingredients to reproduce the real-virtual, abelian-like contribution to the NNLO Drell-Yan K factor. Adding these together according to

$$
K_{\mathrm{rv}}^{(2)}(z)=K_{\mathrm{dB}}^{(2)}(z)+K_{\partial \mathcal{A}}^{(2)}(z)+K_{\text {collinear }}^{(2)}(z),
$$

and using the results for each term given in eqs. (4.18), (4.20), (4.22), we precisely reproduce the full perturbative result, given in eq. (4.7). This is a non-trivial check of the validity of the NLP factorization formula, eq. (3.5), particularly given the fact that all terms in the cross section (including even the constant in the finite part) are reproduced: no complete prediction for single-logarithmic contributions at NLP and NNLO has been given before.

At this point, it is interesting to compare our results with the recent study of ref. [64], which used the method of regions [70-72] to classify the same contributions to the NNLO Drell-Yan K-factor. The diagrams of figure 4 were calculated by expanding the virtual momentum $k_{1}$ in soft, hard and (anti-)collinear regions (the latter with respect to both legs $p$ and $\bar{p}$ ). This involves decomposing the virtual momentum in a Sudakov decomposition, according to

$$
k_{1}^{\mu}=\frac{1}{2}\left(n_{-} \cdot k_{1}\right) n_{+}^{\mu}+\frac{1}{2}\left(n_{+} \cdot k_{1}\right) n_{-}^{\mu}+k_{1 \perp}^{\mu},
$$

where the vectors $n_{ \pm}$were chosen to be parallel to the $p$ and $\bar{p}$ directions, respectively. In fact, they correspond to the vectors $\hat{n}_{i}$ introduced here in section 2 . It was then found that the only contributions to the K-factor originated from the hard and (anti-)collinear regions. The connection with the present study is as follows. The contributions from the hard region consist of the first two terms on the right-hand side of eq. (4.23), namely to the one-loop non-radiative amplitude dressed by an additional emission, and the derivative of the same amplitude. The remaining term in eq. (4.23) comprises contributions from the collinear regions associated with each leg. We see this directly in eq. (3.14), in which the terms on the right-hand side are explicitly proportional to an overall scale factor of $(2 p \cdot k)^{-\epsilon}$ or $(2 \bar{p} \cdot k)^{-\epsilon}$. This is the only scale that survives in the collinear region, and it is precisely these contributions that the original Low's theorem [48] fails to capture, but which are discussed extensively in ref. [50] (see also ref. [96]). Note that the exact correspondence is achieved because we have chosen here the $n_{i}$ vectors in accordance with a method-of-regions, or effective-theory approach.

It is also interesting to examine our results in light of the recently proposed next-to-soft theorems in gravity [79] and gauge theories [80], discussed further in refs. [75-88, 91-94, 97]. First, one may note that the next-to-soft theorems as presented in refs. [79, 80] involve an explicit coupling to the total angular momentum of each outgoing leg, a structure which is not immediately apparent in eq. (2.29). This is ultimately due, however, to the fact that eq. (2.29) is presented in a form which is most straightforward for explicit calculation of the various contributions. Nevertheless, one does see this structure emerge at loop level ${ }^{11}$ in eq. (3.5), where the second and third terms on the right-hand side combine to give

$$
G_{i}^{\nu \mu}\left(\frac{\partial}{\partial p_{i}^{\nu}}+J_{\nu}^{(0)}\right) \mathcal{A}^{(1)}=\frac{\mathrm{i} k_{2}^{\nu}}{p \cdot k_{2}}\left[L_{\mu \nu}^{(i)}+\Sigma_{\mu \nu}^{(i)}\right] \mathcal{A}^{(1)},
$$

\footnotetext{
${ }^{11}$ The tree-level case has already been examined in ref. [97].
} 
where $\Sigma_{\mu \nu}^{(i)}$ is the spin angular momentum associated with leg $i$, defined in eq. (3.8), and

$$
L_{\mu \nu}^{(i)}=x_{i \mu} p_{i \nu}-x_{i \nu} p_{i \mu}=\mathrm{i}\left(p_{i \mu} \frac{\partial}{\partial p_{i}^{\nu}}-p_{i \nu} \frac{\partial}{\partial p_{i}^{\mu}}\right)
$$

is the orbital angular momentum. Eq. (4.25) is precisely the coupling to the total angular momentum observed in ref. [80]. Note, however, that the final term in eq. (3.5) involves a coupling to the spin angular momentum only, and thus corresponds to an explicit breaking of the next-to-soft theorem at loop level. As discussed above, this breaking is associated with collinear effects.

A particular point of discussion in recent literature is whether or not the next-to-soft theorems of refs. $[79,80]$ receive corrections at loop level. This has been related to the sequential order in which one performs the dimensional regularization and soft expansions, with [85] advocating performing the soft expansion first. This was further discussed in refs. [82, 88, 89], with ref. [82] strongly arguing that the expansion in the dimensional regularization parameter $\epsilon$ should be carried out first. A concrete example was examined in ref. [64], namely the real-virtual corrections to the Drell-Yan K factor, that we have also considered here. There, it was found that contributions with a logarithmic dependence on the radiated gluon momentum $k$ are generated in the amplitude from the collinear region. These are needed to reproduce the known $\mathrm{K}$ factor, and would be manifestly absent upon carrying out the soft expansion before the $\epsilon$ expansion. In the present context, this corresponds to expanding in $k$ before carrying out the integration over the virtual gluon momentum $k_{1}$. The presence of such terms indicate that there are indeed loop corrections to next-to-soft theorems.

The results of this paper, building upon the earlier work of ref. [50], provide for the first time a description of this breaking in terms of a universal factorized expression. It is then interesting to trace these loop corrections in the NLP expression for the amplitude, eq. (2.29). As ref. [64] makes clear for the Drell-Yan example, the terms which are problematic from the point of view of the next-to-soft theorems, and which depend upon the ordering of the soft and $\epsilon$ expansions, are those with an overall power of $(p \cdot k)^{-\epsilon}$, or $(\bar{p} \cdot k)^{-\epsilon}$. As already noted above, these stem from the collinear regions associated with the corresponding external legs, and appear here in the second line of eq. (2.29), through the radiative jet function $J_{\mu}$. This is not surprising: the failure of the next-to-soft theorems at loop level means that extra information must be inserted by hand in order to patch up the collinear regions. This explains the presence of the jet emission function, and its definition makes clear that such terms are universal.

\section{Conclusion}

In this paper, we have considered the generalization of the soft-collinear factorization theorem for gauge-theory scattering amplitudes to include corrections which are responsible for next-to-leading power threshold logarithms in high-energy cross sections. We concentrated on electroweak annihilation cross sections, using the Drell-Yan process as an example, since 
in this case final state radiation is forced to be (next-to-) soft near threshold, and the problem of NLP threshold logarithms can in principle be attacked using the tools provided by the Low-Burnett-Kroll-Del Duca theorem. The organization of these logarithms at finite order, and possibly to all orders in perturbation theory, is likely to play a crucial role in improving the precision of collider physics predictions, and it might also have a number of more formal applications, concerning the infrared limit of scattering amplitudes beyond divergent contributions.

Building upon the work of ref. [50], we have constructed an explicit expression for the radiative scattering amplitude, in which a (next-to-) soft gluon is emitted into the final state of the Drell-Yan process. Our basic result is best summarized in eq. (3.3), which expresses NLP corrections to the amplitude in terms of known universal quantities that appear in the soft-collinear factorization formula, as well as a new universal quantity, the radiative jet function, defined in terms of an auxiliary 'factorization vector' $n$ in a manner similar to ordinary (non-radiative) jets. This function first appeared in ref. [50], and is computed here for the first time at the one-loop level. We have also refined the treatment of ref. [50] by introducing the explicit factorization of soft modes, including the correct treatment of the double counting of soft-collinear singularities, and by studying the role played by the factorization vectors $n_{i}$. In particular, we have noted that it is possible, in this case, to work with light-like reference vectors, $n_{i}^{2}=0$, without introducing any further double countings of poles, or other inconsistencies, thanks to the simple renormalization properties of the proposed NLP expression for the amplitude, eq. (3.3). This has led us to a considerably simplified formula, eq. (3.5), in terms of light-like reference vectors, which can be directly applied to construct NLP-accurate cross sections.

In order to test our generalized factorization formula, we have used it to reproduce the known NLP contributions in the abelian-like real-virtual contribution to the DrellYan K-factor at NNLO. Purely real emission (of two gluons) at this order was already understood for this process in ref. [57], using results based only on the soft expansion, pushed to next-to-soft level. It is only when virtual gluons are present that one becomes sensitive to collinear effects. Thus, our exercise is a highly non-trivial check of eq. (3.5), in which all parts of the formula are tested. We find that we do indeed reproduce all NLP threshold contributions, including even the constant term in the finite part, which is not logarithmically enhanced. This suggests that eq. (2.29) can be used even at finite orders to obtain good approximate higher-order cross sections at NNLO and beyond. We emphasize that, while our current results were derived for the abelian color structure, using a QED-like expression for the current, the formalism can be naturally generalized to the full non-abelian theory, by using the non-abelian current in eq. (2.16). When this is done, the process of Higgs production via gluon fusion will become accessible to our treatment, as well as other gluon-initiated cross sections.

Our results are an essential first step to eventually develop a complete resummation formalism for NLP threshold logarithms, but, to this end, much work remains to be done. The next step will be to precisely work out and test the non-abelian version of the present formalism, which will immediately lead to an application to Higgs production in the gluon fusion channel. We will then need to consider in more detail the factorized expressions 
that arise at the level of cross sections, rather than amplitudes, including a fully systematic treatment of multi-gluon phase space. Finally, a non-trivial step will be the generalization to final state jets, which will require a slightly different treatment, due to the importance of hard-collinear gluon contributions, which cannot be reached through the soft expansion. Work on all these issues is in progress.

\section{Acknowledgments}

We thank Pietro Falgari, Gerben Stavenga and Marco Volponi for useful discussions and collaboration during the early stages of this project; we also thank Einan Gardi, Simon Caron-Huot and Duff Neill for more recent discussions. This work was supported by the Research Executive Agency (REA) of the European Union under the Grant Agreements number PITN-GA-2010-264564 (LHCPhenoNet) and PITN-GA-2012-316704 (HIGGSTOOLS); by MIUR (Italy), under contract 2010YJ2NYW_006, and by the University of Torino and the Compagnia di San Paolo under contract ORTO11TPXK. DB and EL have been supported by the Netherlands Foundation for Fundamental Research of Matter (FOM) programme 104, entitled "Theoretical Particle Physics in the Era of the LHC", and the National Organization for Scientific Research (NWO). CDW is supported by the U.K. Science and Technology Facilities Council (STFC). We are grateful to the Higgs Centre for Theoretical Physics at the University of Edinburgh, where part of this work was carried out, for warm hospitality. LM is especially grateful to Nikhef for extended hospitality under the auspices of LHCPhenoNet, and EL to the Munich Institute for Astro- and Particle Physics (MIAPP) of the DFG cluster of excellence "Origin and Structure of the Universe".

\section{A The abelian-like real-virtual NNLO Drell-Yan K-factor}

In eq. (4.7), we quoted a result for the $C_{F}^{2}$ part of the real-virtual contribution to the NNLO Drell-Yan $K$-factor. This has not previously appeared in the literature, so we believe it is useful to collect a few details regarding the calculation. In particular, we show here, in figure 4, the relevant Feynman diagrams for the squared matrix element, and we list below their individual contributions to the cross section at NLP in the threshold expansion. We have carried out the Dirac traces, the integration over the virtual momentum $k_{1}$, and the Passarino-Veltman reduction using FeynCalc [106, 107]. Standard results for oneloop scalar integrals may be taken, for example, from ref. [108]. After substituting the phase space parametrization in eq. (4.5), one may expand the squared amplitude in powers of $(1-z)$, where NLP accuracy corresponds to keeping the first subleading correction. One may then straightforwardly integrate over the real-gluon phase space, using eq. (4.6). Discarding transcendental constants, as in the text, the results for individual Feynman diagrams at NLP accuracy are given by

$$
\begin{aligned}
(a): & \frac{24}{\epsilon^{3}}\left[\mathcal{D}_{0}(z)-1\right]+\frac{8}{\epsilon^{2}}\left[-9 \mathcal{D}_{1}(z)+3 \mathcal{D}_{0}(z)+11 L(z)-11\right] \\
& +\frac{4}{\epsilon}\left[27 \mathcal{D}_{2}(z)-18 \mathcal{D}_{1}(z)+12 \mathcal{D}_{0}(z)-37 L^{2}(z)+68 L(z)-26\right]
\end{aligned}
$$




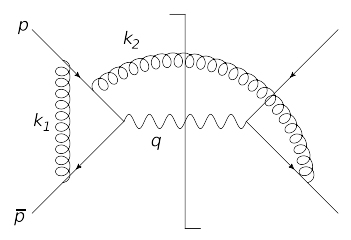

(a)

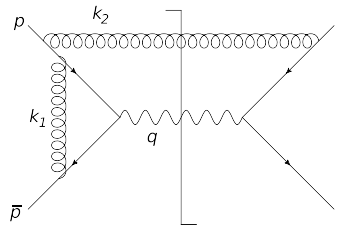

(d)

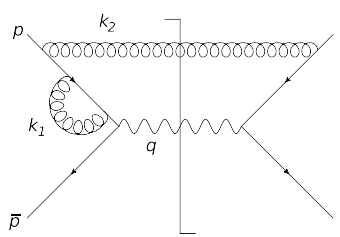

$(g)$

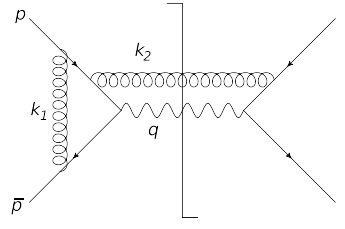

(b)

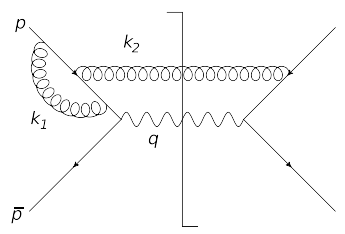

(e)

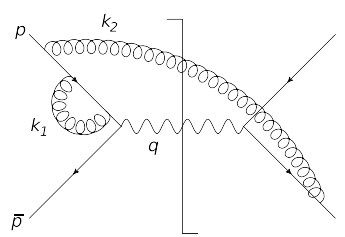

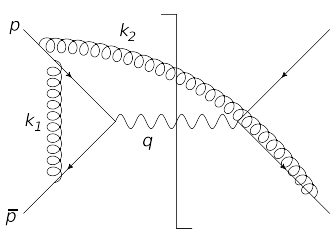

(c)

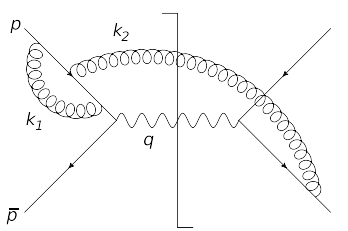

(f)

Figure 4. Feynman diagrams for the matrix element squared contributing to Drell-Yan production at NNLO, and involving one real and one virtual emission. Diagrams obtained by interchanging $p \leftrightarrow \bar{p}$ and/or complex conjugation are not shown.

$$
\begin{aligned}
& -108 \mathcal{D}_{3}(z)+108 \mathcal{D}_{2}(z)-144 \mathcal{D}_{1}(z)+96 \mathcal{D}_{0}(z) \\
& +\frac{476}{3} L^{3}(z)-416 L^{2}(z)+352 L(z)-208
\end{aligned}
$$

(b) : $\quad \frac{8}{\epsilon^{2}}+\frac{8}{\epsilon}[-L(z)+1]-4 L^{2}(z)+32$,

$(c): \quad \frac{8}{\epsilon^{3}}\left[\mathcal{D}_{0}(z)-1\right]+\frac{8}{\epsilon^{2}}\left[\mathcal{D}_{1}(z)+3 \mathcal{D}_{0}(z)-3 L(z)-1\right]$

$$
\begin{aligned}
& +\frac{4}{\epsilon}\left[-11 \mathcal{D}_{2}(z)-6 \mathcal{D}_{1}(z)+20 \mathcal{D}_{0}(z)+21 L^{2}(z)-20 L(z)-20\right] \\
& +\frac{196}{3} \mathcal{D}_{3}(z)-12 \mathcal{D}_{2}(z)-112 \mathcal{D}_{1}(z)+160 \mathcal{D}_{0}(z)-116 L^{3}(z)+224 L^{2}(z)-192,
\end{aligned}
$$

$(d): \quad 0$,

$(e): \frac{4}{\epsilon^{2}}+\frac{4}{\epsilon}[-3 L(z)+1]+18 L^{2}(z)-12 L(z)+8$,

$(f): \quad-\frac{12}{\epsilon^{2}} \mathcal{D}_{0}(z)+\frac{12}{\epsilon}\left[3 \mathcal{D}_{1}(z)-\mathcal{D}_{0}(z)+1\right]$

$$
-54 \mathcal{D}_{2}(z)+36 \mathcal{D}_{1}(z)-24 \mathcal{D}_{0}(z)-36 L(z) \text {, }
$$

$(g): \quad 0$,

$(h): \quad \frac{12}{\epsilon^{2}}\left[\mathcal{D}_{0}(z)-1\right]+\frac{12}{\epsilon}\left[-3 \mathcal{D}_{1}(z)+\mathcal{D}_{0}(z)+3 L(z)-3\right]$

$$
+54 \mathcal{D}_{2}(z)-36 \mathcal{D}_{1}(z)+24 \mathcal{D}_{0}(z)-54 L^{2}(z)+108 L(z)-48,
$$


where we have used the definitions of eq. (4.8), and we have multiplied each diagram by four, in order to take into account complex conjugate diagrams, as well as those obtained from figure 4 by interchanging $p$ and $\bar{p}$.

It is interesting to note that there are eikonal terms in graphs (e)-(h), and they cancel when all such contributions are added together. This had already been noted in ref. [64], and the fact that such terms appear in individual diagrams is an artifact of our (Feynman) gauge choice. Summing all diagrams together, one obtains the result of eq. (4.7). Note also that we have not included UV counterterms in the above calculation: if included, these contributions cancel, in a manner reminiscent of the Ward identity that prevents QCD corrections from renormalising the (QED) photon-fermion vertex.

Open Access. This article is distributed under the terms of the Creative Commons Attribution License (CC-BY 4.0), which permits any use, distribution and reproduction in any medium, provided the original author(s) and source are credited.

\section{References}

[1] G.F. Sterman, Summation of large corrections to short distance hadronic cross-sections, Nucl. Phys. B 281 (1987) 310 [InSPIRE].

[2] S. Catani and L. Trentadue, Resummation of the QCD Perturbative Series for Hard Processes, Nucl. Phys. B 327 (1989) 323 [InSPIRE].

[3] H. Contopanagos, E. Laenen and G.F. Sterman, Sudakov factorization and resummation, Nucl. Phys. B 484 (1997) 303 [hep-ph/9604313] [INSPIRE].

[4] G.P. Korchemsky and G. Marchesini, Resummation of large infrared corrections using Wilson loops, Phys. Lett. B 313 (1993) 433 [INSPIRE].

[5] G.P. Korchemsky and G. Marchesini, Structure function for large $x$ and renormalization of Wilson loop, Nucl. Phys. B 406 (1993) 225 [hep-ph/9210281] [InSPIRE].

[6] S. Forte and G. Ridolfi, Renormalization group approach to soft gluon resummation, Nucl. Phys. B 650 (2003) 229 [hep-ph/0209154] [INSPIRE].

[7] T. Becher and M. Neubert, Threshold resummation in momentum space from effective field theory, Phys. Rev. Lett. 97 (2006) 082001 [hep-ph/0605050] [INSPIRE].

[8] M.D. Schwartz, Resummation and NLO matching of event shapes with effective field theory, Phys. Rev. D 77 (2008) 014026 [arXiv:0709.2709] [INSPIRE].

[9] C.W. Bauer, S.P. Fleming, C. Lee and G.F. Sterman, Factorization of $e^{+} e^{-}$Event Shape Distributions with Hadronic Final States in Soft Collinear Effective Theory, Phys. Rev. D 78 (2008) 034027 [arXiv: 0801.4569] [INSPIRE].

[10] J.-y. Chiu, A. Fuhrer, R. Kelley and A.V. Manohar, Factorization Structure of Gauge Theory Amplitudes and Application to Hard Scattering Processes at the LHC, Phys. Rev. D 80 (2009) 094013 [arXiv: 0909.0012] [INSPIRE].

[11] E. Laenen, G. Stavenga and C.D. White, Path integral approach to eikonal and next-to-eikonal exponentiation, JHEP 03 (2009) 054 [arXiv:0811.2067] [INSPIRE].

[12] J.C. Collins, Sudakov form-factors, Adv. Ser. Direct. High Energy Phys. 5 (1989) 573 [hep-ph/0312336] [INSPIRE]. 
[13] L.J. Dixon, L. Magnea and G.F. Sterman, Universal structure of subleading infrared poles in gauge theory amplitudes, JHEP 08 (2008) 022 [arXiv:0805.3515] [INSPIRE].

[14] S. Moch, J.A.M. Vermaseren and A. Vogt, The quark form-factor at higher orders, JHEP 08 (2005) 049 [hep-ph/0507039] [INSPIRE].

[15] S. Moch, J.A.M. Vermaseren and A. Vogt, Three-loop results for quark and gluon form-factors, Phys. Lett. B 625 (2005) 245 [hep-ph/0508055] [INSPIRE].

[16] S.M. Aybat, L.J. Dixon and G.F. Sterman, The two-loop anomalous dimension matrix for soft gluon exchange, Phys. Rev. Lett. 97 (2006) 072001 [hep-ph/0606254] [INSPIRE].

[17] S.M. Aybat, L.J. Dixon and G.F. Sterman, The two-loop soft anomalous dimension matrix and resummation at next-to-next-to leading pole, Phys. Rev. D 74 (2006) 074004 [hep-ph/0607309] [INSPIRE].

[18] T. Becher and M. Neubert, Infrared singularities of scattering amplitudes in perturbative QCD, Phys. Rev. Lett. 102 (2009) 162001 [arXiv:0901.0722] [InSPIRE].

[19] E. Gardi and L. Magnea, Factorization constraints for soft anomalous dimensions in QCD scattering amplitudes, JHEP 03 (2009) 079 [arXiv:0901.1091] [INSPIRE].

[20] T. Becher and M. Neubert, On the structure of infrared singularities of gauge-theory amplitudes, JHEP 06 (2009) 081 [arXiv: 0903.1126] [INSPIRE].

[21] N. Kidonakis, Two-loop soft anomalous dimensions and NNLL resummation for heavy quark production, Phys. Rev. Lett. 102 (2009) 232003 [arXiv:0903.2561] [INSPIRE].

[22] T. Becher and M. Neubert, Infrared singularities of QCD amplitudes with massive partons, Phys. Rev. D 79 (2009) 125004 [arXiv:0904.1021] [INSPIRE].

[23] A. Ferroglia, M. Neubert, B.D. Pecjak and L.L. Yang, Two-loop divergences of massive scattering amplitudes in non-abelian gauge theories, JHEP 11 (2009) 062 [arXiv:0908.3676] [INSPIRE].

[24] A. Ferroglia, M. Neubert, B.D. Pecjak and L.L. Yang, Two-loop divergences of scattering amplitudes with massive partons, Phys. Rev. Lett. 103 (2009) 201601 [arXiv:0907.4791] [INSPIRE].

[25] A. Mitov, G.F. Sterman and I. Sung, The Massive Soft Anomalous Dimension Matrix at Two Loops, Phys. Rev. D 79 (2009) 094015 [arXiv: 0903.3241] [INSPIRE].

[26] A. Mitov, G. Sterman and I. Sung, Diagrammatic Exponentiation for Products of Wilson Lines, Phys. Rev. D 82 (2010) 096010 [arXiv:1008.0099] [INSPIRE].

[27] E. Gardi, E. Laenen, G. Stavenga and C.D. White, Webs in multiparton scattering using the replica trick, JHEP 11 (2010) 155 [arXiv: 1008.0098] [INSPIRE].

[28] E. Gardi and C.D. White, General properties of multiparton webs: proofs from combinatorics, JHEP 03 (2011) 079 [arXiv: 1102.0756] [INSPIRE].

[29] E. Gardi, J.M. Smillie and C.D. White, On the renormalization of multiparton webs, JHEP 09 (2011) 114 [arXiv:1108.1357] [INSPIRE].

[30] D. Correa, J. Henn, J. Maldacena and A. Sever, The cusp anomalous dimension at three loops and beyond, JHEP 05 (2012) 098 [arXiv: 1203.1019] [INSPIRE].

[31] J.M. Henn and T. Huber, Systematics of the cusp anomalous dimension, JHEP 11 (2012) 058 [arXiv: 1207.2161] [INSPIRE]. 
[32] M. Dukes, E. Gardi, E. Steingrimsson and C.D. White, Web worlds, web-colouring matrices and web-mixing matrices, J. Comb. Theory Ser. A 120 (2013) 1012 [arXiv:1301.6576] [INSPIRE].

[33] J.M. Henn and T. Huber, The four-loop cusp anomalous dimension in $\mathcal{N}=4$ super Yang-Mills and analytic integration techniques for Wilson line integrals, JHEP 09 (2013) 147 [arXiv: 1304.6418] [INSPIRE].

[34] E. Gardi, J.M. Smillie and C.D. White, The non-Abelian exponentiation theorem for multiple Wilson lines, JHEP 06 (2013) 088 [arXiv:1304.7040] [INSPIRE].

[35] M. Dukes, E. Gardi, H. McAslan, D.J. Scott and C.D. White, Webs and posets, JHEP 01 (2014) 024 [arXiv: 1310.3127] [InSPIRE].

[36] E. Gardi, From webs to polylogarithms, JHEP 04 (2014) 044 [arXiv:1310.5268] [INSPIRE].

[37] A. Grozin, J.M. Henn, G.P. Korchemsky and P. Marquard, The $n_{f}$ terms of the three-loop cusp anomalous dimension in QCD, PoS (LL2014) 016 [arXiv: 1406.7828] [INSPIRE].

[38] G. Falcioni, E. Gardi, M. Harley, L. Magnea and C.D. White, Multiple Gluon Exchange Webs, JHEP 10 (2014) 010 [arXiv:1407.3477] [InSPIRE].

[39] A. Grozin, J.M. Henn, G.P. Korchemsky and P. Marquard, Three Loop Cusp Anomalous Dimension in QCD, Phys. Rev. Lett. 114 (2015) 062006 [arXiv:1409.0023] [INSPIRE].

[40] E. Laenen, Resummation for observables at TeV colliders, Pramana 63 (2004) 1225 [INSPIRE].

[41] G. Parisi, Summing large perturbative corrections in QCD, Phys. Lett. B 90 (1980) 295 [INSPIRE].

[42] T.O. Eynck, E. Laenen and L. Magnea, Exponentiation of the Drell-Yan cross-section near partonic threshold in the DIS and MS-bar schemes, JHEP 06 (2003) 057 [hep-ph/0305179] [INSPIRE].

[43] M. Krämer, E. Laenen and M. Spira, Soft gluon radiation in Higgs boson production at the LHC, Nucl. Phys. B 511 (1998) 523 [hep-ph/9611272] [inSPIRE].

[44] R.V. Harlander and W.B. Kilgore, Soft and virtual corrections to $p p \rightarrow H+X$ at NNLO, Phys. Rev. D 64 (2001) 013015 [hep-ph/0102241] [INSPIRE].

[45] R.V. Harlander and W.B. Kilgore, Next-to-next-to-leading order Higgs production at hadron colliders, Phys. Rev. Lett. 88 (2002) 201801 [hep-ph/0201206] [INSPIRE].

[46] S. Catani, D. de Florian and M. Grazzini, Higgs production in hadron collisions: soft and virtual QCD corrections at NNLO, JHEP 05 (2001) 025 [hep-ph/0102227] [INSPIRE].

[47] S. Catani, D. de Florian, M. Grazzini and P. Nason, Soft gluon resummation for Higgs boson production at hadron colliders, JHEP 07 (2003) 028 [hep-ph/0306211] [INSPIRE].

[48] F.E. Low, Bremsstrahlung of very low-energy quanta in elementary particle collisions, Phys. Rev. 110 (1958) 974 [INSPIRE].

[49] T.H. Burnett and N.M. Kroll, Extension of the low soft photon theorem, Phys. Rev. Lett. 20 (1968) 86 [INSPIRE].

[50] V. Del Duca, High-energy bremsstrahlung theorems for soft photons, Nucl. Phys. B 345 (1990) 369 [inSPIRE].

[51] Y. Dokshitzer, G. Marchesini and G.P. Salam, Revisiting parton evolution and the large-x limit, Phys. Lett. B 634 (2006) 504 [hep-ph/0511302] [INSPIRE]. 
[52] E. Laenen, L. Magnea and G. Stavenga, On next-to-eikonal corrections to threshold resummation for the Drell-Yan and DIS cross sections, Phys. Lett. B 669 (2008) 173 [arXiv: 0807.4412] [INSPIRE].

[53] S. Moch and A. Vogt, Threshold Resummation of the Structure Function F(L), JHEP 04 (2009) 081 [arXiv: 0902.2342] [INSPIRE].

[54] G. Grunberg and V. Ravindran, On threshold resummation beyond leading $1-x$ order, JHEP 10 (2009) 055 [arXiv: 0902.2702] [INSPIRE].

[55] S. Moch and A. Vogt, On non-singlet physical evolution kernels and large-x coefficient functions in perturbative QCD, JHEP 11 (2009) 099 [arXiv:0909.2124] [INSPIRE].

[56] G. Soar, S. Moch, J.A.M. Vermaseren and A. Vogt, On Higgs-exchange DIS, physical evolution kernels and fourth-order splitting functions at large $x$, Nucl. Phys. B 832 (2010) 152 [arXiv: 0912.0369] [INSPIRE].

[57] E. Laenen, L. Magnea, G. Stavenga and C.D. White, Next-to-eikonal corrections to soft gluon radiation: a diagrammatic approach, JHEP 01 (2011) 141 [arXiv:1010.1860] [INSPIRE].

[58] A.A. Almasy, G. Soar and A. Vogt, Generalized double-logarithmic large-x resummation in inclusive deep-inelastic scattering, JHEP 03 (2011) 030 [arXiv:1012.3352] [INSPIRE].

[59] R.D. Ball, M. Bonvini, S. Forte, S. Marzani and G. Ridolfi, Higgs production in gluon fusion beyond NNLO, Nucl. Phys. B 874 (2013) 746 [arXiv:1303.3590] [InSPIRE].

[60] T. Altinoluk, N. Armesto, G. Beuf, M. Martínez and C.A. Salgado, Next-to-eikonal corrections in the CGC: gluon production and spin asymmetries in $p A$ collisions, JHEP 07 (2014) 068 [arXiv: 1404.2219] [INSPIRE].

[61] L. Apolinário, N. Armesto, J.G. Milhano and C.A. Salgado, Medium-induced gluon radiation and colour decoherence beyond the soft approximation, JHEP 02 (2015) 119 [arXiv: 1407.0599] [INSPIRE].

[62] D. de Florian, J. Mazzitelli, S. Moch and A. Vogt, Approximate $N^{3}$ LO Higgs-boson production cross section using physical-kernel constraints, JHEP 10 (2014) 176 [arXiv: 1408.6277] [INSPIRE].

[63] N.A. Lo Presti, A.A. Almasy and A. Vogt, Leading large-x logarithms of the quark-gluon contributions to inclusive Higgs-boson and lepton-pair production, Phys. Lett. B 737 (2014) 120 [arXiv: 1407.1553] [INSPIRE].

[64] D. Bonocore, E. Laenen, L. Magnea, L. Vernazza and C.D. White, The method of regions and next-to-soft corrections in Drell-Yan production, Phys. Lett. B 742 (2015) 375 [arXiv: 1410.6406] [INSPIRE].

[65] T. Matsuura, S.C. van der Marck and W.L. van Neerven, The Order $\alpha_{s}^{2}$ Contribution to the K Factor of the Drell-Yan Process, Phys. Lett. B 211 (1988) 171 [INSPIRE].

[66] T. Matsuura, S.C. van der Marck and W.L. van Neerven, The Calculation of the Second Order Soft and Virtual Contributions to the Drell-Yan Cross-Section, Nucl. Phys. B 319 (1989) 570 [INSPIRE].

[67] T. Matsuura and W.L. van Neerven, Second order logarithmic corrections to the Drell-Yan cross-section, Z. Phys. C 38 (1988) 623 [inSPIRE].

[68] R. Hamberg, W.L. van Neerven and T. Matsuura, A complete calculation of the order $\alpha_{s}^{2}$ correction to the Drell-Yan K factor, Nucl. Phys. B 359 (1991) 343 [INSPIRE]. 
[69] T. Matsuura, R. Hamberg and W.L. van Neerven, The contribution of the gluon gluon subprocess to the Drell-Yan K-factor, Nucl. Phys. Proc. Suppl. 23B (1991) 3 [InSPIRE].

[70] M. Beneke and V.A. Smirnov, Asymptotic expansion of Feynman integrals near threshold, Nucl. Phys. B 522 (1998) 321 [hep-ph/9711391] [INSPIRE].

[71] A. Pak and A. Smirnov, Geometric approach to asymptotic expansion of Feynman integrals, Eur. Phys. J. C 71 (2011) 1626 [arXiv:1011.4863] [InSPIRE].

[72] B. Jantzen, Foundation and generalization of the expansion by regions, JHEP 12 (2011) 076 [arXiv:1111.2589] [INSPIRE].

[73] C. Anastasiou, C. Duhr, F. Dulat, F. Herzog and B. Mistlberger, Real-virtual contributions to the inclusive Higgs cross-section at $N^{3} L O$, JHEP 12 (2013) 088 [arXiv:1311.1425] [INSPIRE].

[74] F. Herzog and B. Mistlberger, The Soft-Virtual Higgs Cross-section at N3LO and the Convergence of the Threshold Expansion, arXiv:1405.5685 [INSPIRE].

[75] F. Cachazo, S. He and E.Y. Yuan, Scattering of Massless Particles in Arbitrary Dimensions, Phys. Rev. Lett. 113 (2014) 171601 [arXiv:1307.2199] [InSPIRE].

[76] F. Cachazo, S. He and E.Y. Yuan, Scattering of massless particles: scalars, gluons and gravitons, JHEP 07 (2014) 033 [arXiv: 1309.0885] [INSPIRE].

[77] A. Strominger, On BMS invariance of gravitational scattering, JHEP 07 (2014) 152 [arXiv: 1312.2229] [INSPIRE].

[78] T. He, V. Lysov, P. Mitra and A. Strominger, BMS supertranslations and Weinberg's soft graviton theorem, arXiv:1401.7026 [INSPIRE].

[79] F. Cachazo and A. Strominger, Evidence for a New Soft Graviton Theorem, arXiv: 1404.4091 [INSPIRE].

[80] E. Casali, Soft sub-leading divergences in Yang-Mills amplitudes, JHEP 08 (2014) 077 [arXiv: 1404.5551] [INSPIRE].

[81] B.U.W. Schwab and A. Volovich, Subleading soft theorem in arbitrary dimensions from scattering equations, Phys. Rev. Lett. 113 (2014) 101601 [arXiv:1404.7749] [INSPIRE].

[82] Z. Bern, S. Davies and J. Nohle, On Loop Corrections to Subleading Soft Behavior of Gluons and Gravitons, Phys. Rev. D 90 (2014) 085015 [arXiv:1405.1015] [INSPIRE].

[83] S. He, Y.-t. Huang and C. Wen, Loop corrections to soft theorems in gauge theories and gravity, JHEP 12 (2014) 115 [arXiv: 1405.1410] [INSPIRE].

[84] A.J. Larkoski, Conformal Invariance of the Subleading Soft Theorem in Gauge Theory, Phys. Rev. D 90 (2014) 087701 [arXiv:1405.2346] [INSPIRE].

[85] F. Cachazo and E.Y. Yuan, Are Soft Theorems Renormalized?, arXiv:1405.3413 [inSPIRE].

[86] N. Afkhami-Jeddi, Soft Graviton Theorem in Arbitrary Dimensions, arXiv:1405.3533 [INSPIRE].

[87] T. Adamo, E. Casali and D. Skinner, Perturbative gravity at null infinity, Class. Quant. Grav. 31 (2014) 225008 [arXiv: 1405.5122] [INSPIRE].

[88] M. Bianchi, S. He, Y.-t. Huang and C. Wen, More on Soft Theorems: Trees, Loops and Strings, arXiv: 1406.5155 [INSPIRE].

[89] Z. Bern, S. Davies, P. Di Vecchia and J. Nohle, Low-Energy Behavior of Gluons and Gravitons from Gauge Invariance, Phys. Rev. D 90 (2014) 084035 [arXiv:1406.6987] [INSPIRE]. 
[90] J. Broedel, M. de Leeuw, J. Plefka and M. Rosso, Constraining subleading soft gluon and graviton theorems, Phys. Rev. D 90 (2014) 065024 [arXiv:1406.6574] [InSPIRE].

[91] T. He, P. Mitra, A.P. Porfyriadis and A. Strominger, New symmetries of massless QED, JHEP 10 (2014) 112 [arXiv:1407.3789] [INSPIRE].

[92] M. Zlotnikov, Sub-sub-leading soft-graviton theorem in arbitrary dimension, JHEP 10 (2014) 148 [arXiv: 1407.5936] [INSPIRE].

[93] C. Kalousios and F. Rojas, Next to subleading soft-graviton theorem in arbitrary dimensions, JHEP 01 (2015) 107 [arXiv:1407.5982] [INSPIRE].

[94] Y.-J. Du, B. Feng, C.-H. Fu and Y. Wang, Note on soft graviton theorem by KLT relation, JHEP 11 (2014) 090 [arXiv: 1408.4179] [INSPIRE].

[95] H. Lüo, P. Mastrolia and W.J. Torres Bobadilla, Subleading soft behavior of QCD amplitudes, Phys. Rev. D 91 (2015) 065018 [arXiv:1411.1669] [INSPIRE].

[96] A.J. Larkoski, D. Neill and I.W. Stewart, Soft Theorems from Effective Field Theory, arXiv: 1412.3108 [INSPIRE].

[97] C.D. White, Diagrammatic insights into next-to-soft corrections, Phys. Lett. B 737 (2014) 216 [arXiv: 1406.7184] [INSPIRE].

[98] C.D. White, Factorization properties of soft graviton amplitudes, JHEP 05 (2011) 060 [arXiv:1103.2981] [INSPIRE].

[99] E. Gardi and L. Magnea, Infrared singularities in QCD amplitudes, Nuovo Cim. C32N5-6 (2009) 137 [arXiv: 0908.3273] [INSPIRE].

[100] S. Weinberg, The quantum theory of fields. Vol. 2: Modern applications, Cambridge University Press, Cambridge U.K. (2005).

[101] J. Grammer, G. and D.R. Yennie, Improved treatment for the infrared divergence problem in quantum electrodynamics, Phys. Rev. D 8 (1973) 4332 [INSPIRE].

[102] R. Hamberg, W.L. van Neerven and T. Matsuura, A complete calculation of the order $\alpha_{s}^{2}$ correction to the Drell-Yan K factor, Nucl. Phys. B 359 (1991) 343 [Erratum ibid. B 644 (2002) 403] [INSPIRE].

[103] T. Matsuura, S.C. van der Marck and W.L. van Neerven, The Calculation of the Second Order Soft and Virtual Contributions to the Drell-Yan Cross-Section, Nucl. Phys. B 319 (1989) 570 [inSPIRE].

[104] Y. Li, A. von Manteuffel, R.M. Schabinger and H.X. Zhu, $N^{3}$ LO Higgs boson and Drell-Yan production at threshold: The one-loop two-emission contribution, Phys. Rev. D 90 (2014) 053006 [arXiv: 1404.5839] [INSPIRE].

[105] Y. Li, A. von Manteuffel, R.M. Schabinger and H.X. Zhu, Soft-virtual corrections to Higgs production at $N^{3} L O$, Phys. Rev. D 91 (2015) 036008 [arXiv:1412.2771] [INSPIRE].

[106] J. Kublbeck, H. Eck and R. Mertig, Computeralgebraic generation and calculation of Feynman graphs using FeynArts and FeynCalc, Nucl. Phys. Proc. Suppl. 29A (1992) 204 [INSPIRE].

[107] G. Passarino and M.J.G. Veltman, One Loop Corrections for $e^{+} e^{-}$Annihilation Into $\mu^{+} \mu^{-}$ in the Weinberg Model, Nucl. Phys. B 160 (1979) 151 [INSPIRE].

[108] R.K. Ellis and G. Zanderighi, Scalar one-loop integrals for QCD, JHEP 02 (2008) 002 [arXiv:0712.1851] [INSPIRE]. 